ARTICLE

\title{
BK channel inactivation gates daytime excitability in the circadian clock
}

Joshua P. Whitt ${ }^{1}$, Jenna R. Montgomery ${ }^{1, \dagger} \&$ Andrea L. Meredith ${ }^{1}$

Inactivation is an intrinsic property of several voltage-dependent ion channels, closing the conduction pathway during membrane depolarization and dynamically regulating neuronal activity. $\mathrm{BK} \mathrm{K}^{+}$channels undergo $\mathrm{N}$-type inactivation via their $\beta 2$ subunit, but the physiological significance is not clear. Here, we report that inactivating BK currents predominate during the day in the suprachiasmatic nucleus, the brain's intrinsic clock circuit, reducing steady-state current levels. At night inactivation is diminished, resulting in larger BK currents. Loss of $\beta 2$ eliminates inactivation, abolishing the diurnal variation in both BK current magnitude and SCN firing, and disrupting behavioural rhythmicity. Selective restoration of inactivation via the $\beta 2 \mathrm{~N}$-terminal 'ball-and-chain' domain rescues BK current levels and firing rate, unexpectedly contributing to the subthreshold membrane properties that shift SCN neurons into the daytime 'upstate'. Our study reveals the clock employs inactivation gating as a biophysical switch to set the diurnal variation in suprachiasmatic nucleus excitability that underlies circadian rhythm.

\footnotetext{
${ }^{1}$ Department of Physiology, University of Maryland School of Medicine, Baltimore, Maryland 21201, USA. † Present address: Takeda Cambridge Ltd., Cambridge CB4 OPA, UK. Correspondence and requests for materials should be addressed to A.L.M. (email: ameredith@som.umaryland.edu).
} 
nactivation gating of ion channels is essential to electrical signalling in most neurons. First characterized in Hodgkin and Huxley's classic studies of the action potential ${ }^{1}$, since then, several distinct inactivation mechanisms have been identified in $\mathrm{Na}^{+}, \mathrm{Ca}^{2+}$ and $\mathrm{K}^{+}$channels ${ }^{2-5}$. Among voltage-gated $\mathrm{K}^{+}$ channels, inactivation can occur via the classic 'ball-and-chain' mechanism involving pore occlusion by an intrinsic N-terminal 'ball' located on the cytosolic side of the $\alpha$ pore-forming subunit ${ }^{6}$. In contrast, large conductance $\mathrm{Ca}^{2+}{ }_{-}$and voltage-activated $\mathrm{BK} \mathrm{K}^{+}$channels $\left(K_{\mathrm{Ca}} 1.1, K C N M A 1\right)$ are not intrinsically inactivating ${ }^{7,8}$. However, inactivating $\mathrm{BK}$ currents have been observed in some central neurons, sensory neurons and neuroendocrine cells ${ }^{9-17}$. In adrenal chromaffin cells, BK inactivation is mediated by the $\beta 2$ subunit $^{18}$ and shares several common features with Shaker N-type inactivation, including a rapid time course and contribution of intracellular domains sensitive to proteolytic cleavage ${ }^{19}$. Chromaffin cells express both inactivating and non-inactivating BK channels, associated with distinct action potential behaviour ${ }^{20}$. Yet despite the welldescribed mechanistic basis, understanding the physiological relevance for $\mathrm{BK}$ inactivation has been complicated by other properties conferred by the $\beta 2$ subunit, including a shift in the voltage dependence of activation and slowing of activation and deactivation kinetics ${ }^{18,21}$.

To understand the role of BK channel inactivation in neuronal excitability, we identified a circuit where $\beta 2$ is expressed ${ }^{22}$ and where dynamic regulation of the $\mathrm{BK}$ current is critical for neural coding, the suprachiasmatic nucleus $(\mathrm{SCN})$ of the hypothalamus ${ }^{23-25}$. The SCN circuit undergoes synchronized daily oscillations in action potential firing frequency ${ }^{26}$, and circadian behavioural and physiological characteristics are established by the parameters of the SCN circuit rhythm ${ }^{27,28}$. BK channel expression varies over the circadian cycle in both mouse SCN and fly clock neurons ${ }^{24,29-31}$. The daily rhythm in BK protein abundance is linked to Per2 (ref. 24), a component of the canonical transcription-translation feedback loop that encodes circadian time ${ }^{32}$. Correlated with expression levels, SCN neurons exhibit a diurnal difference in steady-state BK current magnitude, with smaller currents recorded during the day and larger currents at night ${ }^{23,24,31}$. Both loss of BK current at night and aberrant increase in BK current during the day result in disrupted circuit rhythmicity, establishing the diurnal variation in $\mathrm{BK}$ current as essential for expressing behavioural rhythms ${ }^{23-}$ $25,29,33$. Loss of this diurnal rhythm in BK current has been recently linked to degradation of circadian rhythm in aged animals ${ }^{33}$, underscoring the significance of understanding the mechanisms that drive rhythms in BK channel activity in SCN.

Here we report that the $\beta 2$ subunit produces inactivation of SCN BK channels. Using patch-clamp, long-term action potential, and behavioural recordings, we show that loss of $\beta 2$ $(\beta 2 \mathrm{KO})$ abolishes the diurnal variation in both $\mathrm{BK}$ current magnitude and SCN firing, and disrupts circadian circuit and locomotor rhythmicity. We link these changes in excitability specifically to $\mathrm{BK}$ channel inactivation by taking advantage of the modular nature of $\beta 2$, which allows selective rescue of inactivation via delivery of the isolated $\beta 2 \mathrm{~N}$-terminal 'ball-and-chain' domain. We further reveal that neurons with inactivating BK currents fire faster, have depolarized membrane potentials and increased input resistance, demonstrating that inactivation unexpectedly contributes to the daytime 'upstate' in SCN neurons by controlling the amount of subthreshold BK current during the inter-spike interval. Thus, inactivation underlies the shift in BK's influence on excitability between day and night, and regulation of this biophysical mechanism is a central node for the circadian regulation of firing in the SCN clock circuit.

\section{Results}

B2 regulates SCN circuit rhythmicity and circadian behaviour. The SCN expresses two subunits with the potential to modify BK channel properties, $\beta 2$ and $\beta 4$ (ref. 22), but of these, only $\beta 2$ can cause inactivation of $\mathrm{BK}$ currents ${ }^{7,8}$. Since $\beta$ subunits are proposed to tailor BK channel properties and contribute to distinct patterns of excitability across tissues ${ }^{34}$, to establish the importance of the $\beta 2$ subunit in the SCN circuit, we made longterm recordings of spontaneous action potential activity from wild-type (WT) and $\beta 2 \mathrm{KO}$ SCNs. Individual SCN neurons function as coupled, autonomous oscillators, generating a robust synchronized rhythm in firing in ex vivo cultures (Fig. 1a) ${ }^{28,35} . \beta 2$ KO SCNs had significantly reduced rhythmicity overall (Fig. 1b). This was due to both a decrease in the number of rhythmic recordings, as well as a reduction in the circadian amplitude of the rhythmic recordings (Fig. 1c,d) ${ }^{36}$. The circadian period of the rhythmic activity was not different (WT, $24.2 \pm 1.3 \mathrm{~h}$ and $\beta 2$ $\mathrm{KO}, 23.4 \pm 1.2 \mathrm{~h}, P=0.8$ ), instead, the reduced circadian amplitude stemmed from decreased action potential activity at the peak (Fig. 1e).

Alterations in the SCN circuit were correlated with disrupted circadian behaviour (Fig. 1f-g). Like SCN circuit activity, circadian behavioural amplitudes were reduced in $\beta 2 \mathrm{KO}$ mice (Fig. 1h; Supplementary Table 1). Decreased circadian behavioural amplitudes are associated with a more labile pacemaker $23,37,38$, and consistent with this, $\beta 2 \mathrm{KO}$ mice re-entrained about a day faster to a $6-\mathrm{h}$ phase advance of the light:dark cycle and had augmented responses to a phase-shifting light pulse (Fig. 1i,j; Supplementary Fig. 1; Supplementary Table 1). Taken together, these results suggest that $\beta 2$ is important for the circadian patterning of action potential activity in the SCN circuit, and that this mechanism is required for proper circadian rhythmicity in the animal as a whole. Furthermore, the circadian alterations were specific to loss of $\beta 2$, as deletion of the $\beta 4$ subunit, which is also expressed in $\mathrm{SCN}^{22}$, did not result in significant changes in neuronal firing or behavioural activity (Fig. 1c-e; Supplementary Table 1; Supplementary Discussion).

B2 mediates BK current inactivation in SCN neurons. To determine the functional relevance of $\beta 2$ regulation of SCN circuit rhythmicity, we recorded BK currents from SCN neurons using whole-cell voltage clamp. We observed two types of macroscopic BK currents which varied by the amount of current decay observed during the voltage step (Fig. 2a). Currents that exhibited a pronounced decay within $30 \mathrm{~ms}$ were termed $\mathrm{BK}_{\mathrm{i}}$ and had a fractional current ratio $\left(I_{\mathrm{BK}} / I_{\mathrm{BKpeak}}\right)<0.7$ at $+90 \mathrm{mV}$ (Fig. 1b; Supplementary Fig. 2a,b). These $\mathrm{BK}_{\mathrm{i}}$ currents decayed with time constants of $44 \pm 4 \mathrm{~ms}$ (at $+90 \mathrm{mV}, n=13$ ) (Fig. 2c; Supplementary Fig. 2c,d), similar to inactivating BK currents described in other neuronal types ${ }^{18,20}$. Other currents were relatively sustained with little decay, termed $\mathrm{BK}_{\mathrm{s}}$ $\left(I_{\mathrm{BK}} / I_{\mathrm{BKpeak}}>0.7\right)$. The much slower time course of decay for $\mathrm{BK}_{\mathrm{s}}$ currents (172 $\pm 13 \mathrm{~ms}, n=17$; Fig. 2c; Supplementary Fig. 2c) is consistent with non-inactivating $\mathrm{BK}$ currents that show a limited attenuation of current due to $\mathrm{Ca}^{2}+$ clearance $^{18}$. Overall, during the day, most currents were $\mathrm{BK}_{\mathrm{i}}$, while at night, most currents were $\mathrm{BK}_{\mathrm{s}}$ (Fig. 2d). This suggested that the number of neurons exhibiting $\mathrm{BK}_{\mathrm{i}}$ and $\mathrm{BK}_{\mathrm{s}}$ currents between day and night SCNs was responsible for the diurnal difference observed in the steady-state BK current magnitude within SCN (Fig. 2e).

Supporting this idea, we did not find that daytime $\mathrm{BK}_{\mathrm{i}}$ currents decayed more rapidly, and thus to a lower fractional current value, compared with night time $\mathrm{BK}_{\mathrm{i}}$ currents. Instead, the average $\mathrm{BK}_{\mathrm{i}}$ current density (Fig. $2 \mathrm{f}$ ) and fractional current values (Supplementary Fig. 2b) were similar between day and night. 


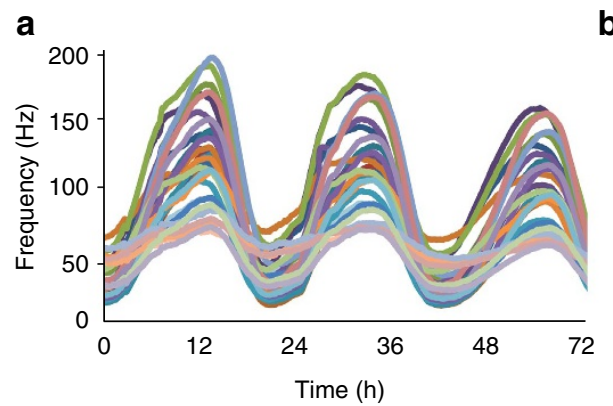

b

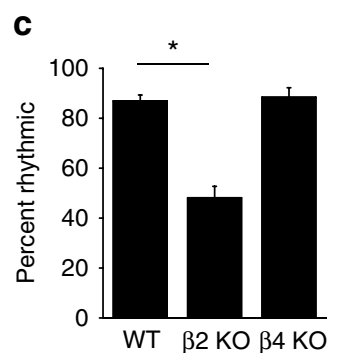

d

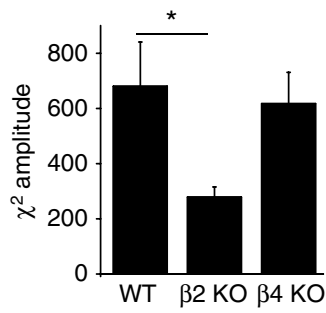

e

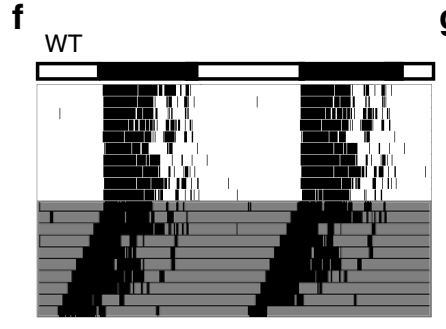

g $\beta 2 \mathrm{KO}$

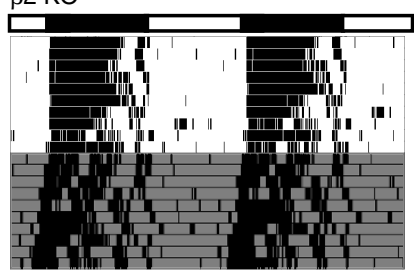

$\mathbf{h}$
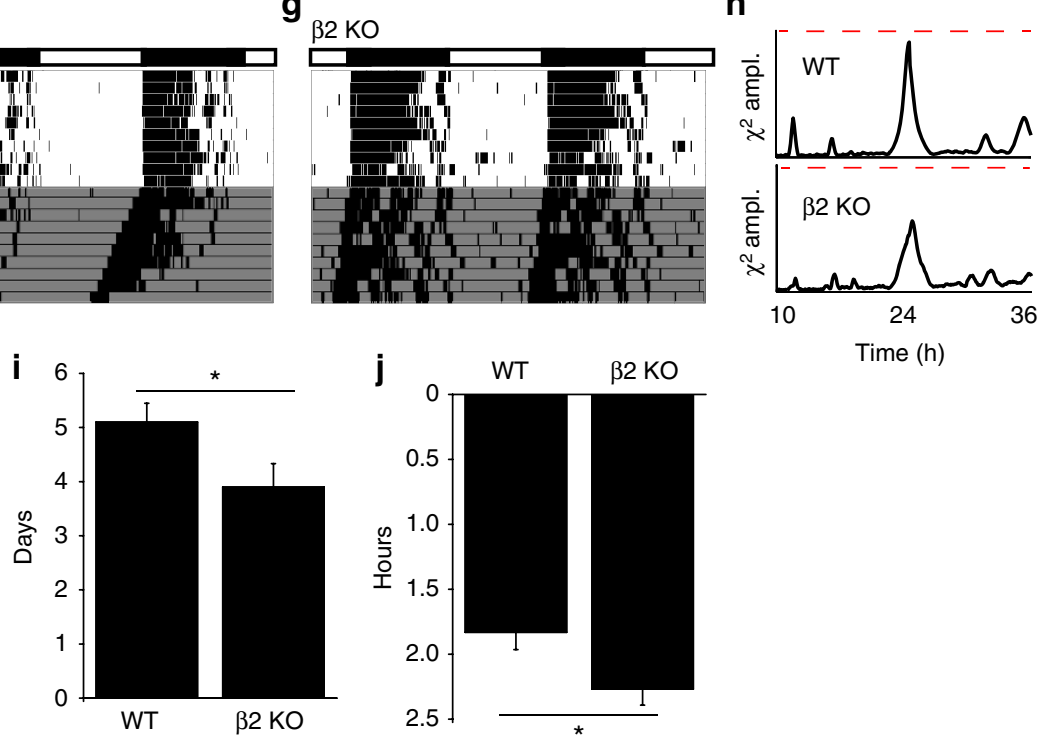

Figure 1 | The $\mathbf{\beta 2}$ subunit is required for SCN neuronal firing rhythmicity and circadian behaviour. (a) Representative spontaneous action potential activity recorded for 3 days on a multielectrode array from WT SCNs. Firing rate shows a robust peak-to-trough difference. (b) $\beta 2 \mathrm{KO}$ SCN activity. Firing amplitude and rhythmicity were reduced. (c) The percentage of recordings within the SCN exhibiting rhythmic firing is decreased in $\beta 2 \mathrm{KO}$ compared with WT and $\beta 4 \mathrm{KO} S \mathrm{SNs}\left(n=8,11\right.$ and $10 \mathrm{SCN}$ slices, respectively). (d) $\chi^{2}$ periodogram analysis of action potential activity. $\chi^{2}$ circadian peak amplitudes were reduced in $\beta 2 \mathrm{KO}$ compared with WT and $\beta 4 \mathrm{KO} S C N s$. (e) Multiunit firing frequency from the peak and trough of rhythmic recordings, or from arrhythmic recordings. $\beta 2 \mathrm{KO}$ firing is reduced during the peak. (f) Locomotor wheel running activity from a representative WT mouse. (g) $\beta 2 \mathrm{KO}$ actogram. (h) $\chi^{2}$ periodogram analysis of wheel behaviour. Dotted line denotes 3,000 (amplitude). (i) $\beta 2 \mathrm{KO}$ mice re-entrained to a $6 \mathrm{~h}$ phase advance of the light-dark cycle faster than WT. (j) Exposure to a light pulse at CT16 caused a greater phase delay in $\beta 2$ KO compared with WT. Representative actograms for $\mathbf{i}$ and $\mathbf{j}$ are in Supplementary Fig. 1. All values are mean \pm s.e.m. ${ }^{\star} P<0.05$, Bonferroni post hoc $(\mathbf{c}-\mathbf{e})$ or $t$-test $(\mathbf{i}-\mathbf{j})$.

These observations support a model where the simple summation of $\mathrm{BK}_{\mathrm{i}}$ to $\mathrm{BK}_{\mathrm{s}}$ currents is a central factor in the day versus night BK current levels observed across the SCN.

Since the $\beta 2$ subunit produces inactivation of $\mathrm{BK}$ channels expressed in heterologous cells and native tissues $7,8,18,34,39$, we examined $\beta 2$ levels to determine whether expression of the $\beta 2$ subunit was correlated with the increased number of $\mathrm{BK}_{\mathrm{i}}$ currents observed in daytime SCNs. BK complexes were immunoprecipitated from SCNs harvested across the circadian cycle. Although the expression of $\beta 2$ did not differ across time points (Fig. $3 \mathrm{a}, \mathrm{b}$ ), the relative ratio of $\beta 2$ associated with the
BK $\alpha$ subunit was higher during the day (Fig. 3c), correlated with the higher proportion of $\mathrm{BK}_{\mathrm{i}}$ currents (Fig. 2d). At night as $\alpha$ expression increased, the ratio of $\beta 2: \alpha$ was lower and consistent with the increased number of $\mathrm{BK}_{\mathrm{s}}$ currents.

To determine whether $\beta 2$ was required for the macroscopic $\mathrm{BK}_{\mathrm{i}}$ current decay in $\mathrm{SCN}$, we recorded $\mathrm{BK}$ currents from $\beta 2 \mathrm{KO}$ neurons. Only $\mathrm{BK}_{\mathrm{s}}$ currents were observed in $\beta 2 \mathrm{KO}$ neurons (Fig. 4a,b; Supplementary Fig. 2a), implicating inactivation as the mechanism for the decay of $\mathrm{BK}_{\mathrm{i}}$ currents. As a consequence of converting all currents to $\mathrm{BK}_{\mathrm{s}}$ during the day, the diurnal difference in $\mathrm{BK}$ current magnitude was abolished in $\beta 2 \mathrm{KO}$ 
a

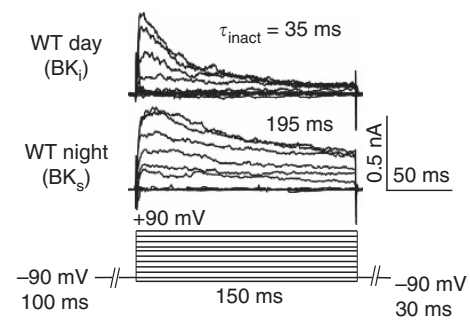

b

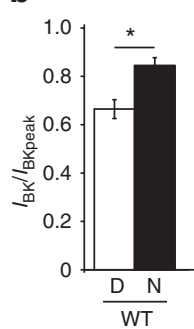

C

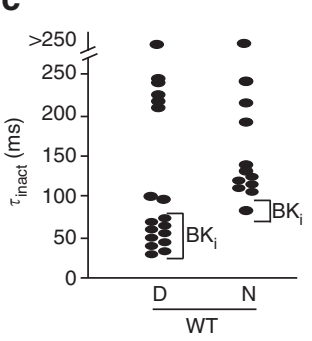

d

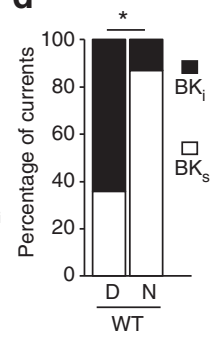

e

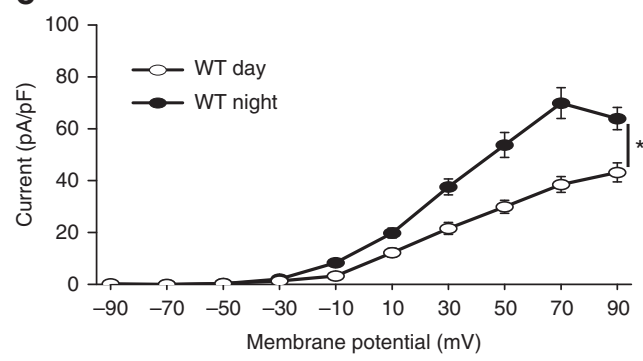

f

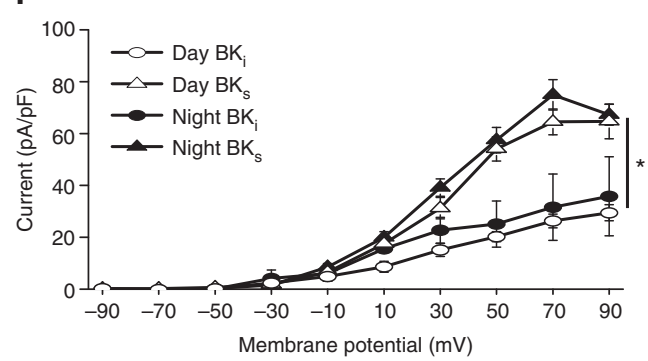

Figure $2 \mid \mathbf{B K}_{\mathbf{i}}$ and $\mathbf{B K} \mathbf{B}_{\mathbf{s}}$ currents in SCN neurons. (a) Representative BK current traces and macroscopic decay time constants $\left(\tau_{i n a c t}\right)$ for $B K_{i}$ and $B K_{s}$ currents from WT SCN neurons. BK currents were isolated by subtraction with the antagonist paxilline (Methods). (b) Fractional BK current $\left(I_{30 m s} / I_{\text {peak }}\right.$ at $+90 \mathrm{mV}$ ) was reduced during the day compared to night in WT neurons, $\mathrm{D}$, daytime recording; $\mathrm{N}$, night time recording. (c) $\tau_{\text {inact }}$ (at $+90 \mathrm{mV}$ ) is lower for daytime $\mathrm{BK}_{\mathrm{i}}$ currents compared to night $\mathrm{BK}_{\mathrm{s}}$ currents in WT neurons. (d) Proportion of neurons with $\mathrm{BK}_{\mathrm{i}}$ and $\mathrm{BK}_{\mathrm{s}}$ currents. WT neurons have more $\mathrm{BK}_{\mathrm{i}}$ currents during the day compared with night. (e) Current density versus voltage relationship for day and night BK currents from WT SCN neurons. The average daytime current magnitude is reduced compared with night. (f) $B K_{i}$ current density is lower than $\mathrm{BK}_{\mathrm{s}}$, during the day or at night. All values are mean \pm s.e.m. $n$ values: $W T$, day $B K_{i}(18), B K_{s}(9)$ and night $B K_{i}(3) B K_{s}(19) .{ }^{\star} P<0.05$, Fisher's exact test (d) or Bonferroni post hoc (e,f).

SCNs (Fig. 4c). Although the small number of $\mathrm{BK}_{\mathrm{i}}$ currents observed in WT neurons at night were also eliminated, the overall night time $\beta 2 \mathrm{KO} B K$ current levels remained unchanged. This was expected since the vast majority of currents were already identified as $\mathrm{BK}_{\mathrm{s}}$ in WT neurons at night. Further consistent with loss of inactivation, the fractional current values and macroscopic current decay time constants were increased in $\beta 2 \mathrm{KO}$ during the day, with no change at night (Fig. 4d,e; Supplementary Fig. 2a-c). These results demonstrate that $\beta 2$ underlies the reduction in steady-state BK current levels during the day and is thereby the central regulator of the diurnal difference in SCN BK current. As a control, we also assayed the expression level and diurnal pattern of the BK channel $\alpha$ subunit in $\beta 2 \mathrm{KO}$ SCNs and found no difference (Supplementary Fig. $4 \mathrm{a}-\mathrm{c}$ ), corroborating the alterations in BK current magnitude stemmed from a loss of $\beta 2$ function and not alteration of BK channel trafficking.

$\beta$ Subunits produce complex effects on BK channel gating, including slowing activation and deactivation, and shifting the voltage dependence of activation to more hyperpolarized potentials $s^{7,8,34,39}$. However, $\beta 2$-mediated inactivation is separably mediated by the intracellular $\mathrm{N}$ terminus, and transplantation or exogenous application of this domain confers inactivation gating to the BK $\alpha$ subunit in the absence of the $\beta 2$ subunit ${ }^{7,11,40,41}$. Conversely, mutation of the three residues comprising the hydrophobic 'ball' $(\Delta$ FIW) removes inactivation while leaving other gating properties of $\beta 2$ intact $^{42}$. If inactivation is the major determinant of the day versus night difference in steady-state current levels, then selective restoration of inactivation during the day with the isolated $\mathrm{N}$ terminus should restore this difference to $\beta 2 \mathrm{KO}$ neurons. Alternatively, failure to restore the decreased daytime BK current levels via inactivation with the $\beta 2 \mathrm{~N}$ terminus would suggest that other properties conferred by the $\beta 2$ subunit are necessary for the daytime decrease in steady-state BK current levels.

To test this, we first delivered the $\beta 2 \mathrm{~N}$ terminus $(\beta 2 \mathrm{~N})$ as a soluble peptide to $\mathrm{BK}$ channels expressed in heterologous
HEK293 cells (Fig. 5). $\quad \beta 2 \mathrm{~N}$ produced a dose-dependent inactivation, while a peptide harbouring the FIW mutation produced no effect $\left(\beta 2 \mathrm{~N}^{\Delta \text { FIW }}\right.$; Fig. $\left.5 \mathrm{a}-\mathrm{d}\right)$. The voltage dependence of inactivation with $\beta 2 \mathrm{~N}$ was similar to the intact $\beta 2$ subunit (Fig. 5e), and $\tau_{\text {inact }}$ values at $\beta 2 \mathrm{~N}$ concentrations above $50 \mu \mathrm{M}$ were similar to $\mathrm{WT} \mathrm{BK}_{\mathrm{i}}$ currents recorded during the day from SCN neurons (Fig. $5 \mathrm{~d}$ ). We next applied the $\beta 2 \mathrm{~N}$ peptide to SCN neurons via the patch pipette.

In $\beta 2 \mathrm{KO}$ SCN neurons, application of $\beta 2 \mathrm{~N}$, but not $\beta 2 \mathrm{~N}^{\Delta \mathrm{FIW}}$, restored the macroscopic decay and reduced the fractional current (Fig. 6a; Supplementary Fig. 2a). The voltage dependence of inactivation and $\tau_{\text {inact }}$ values with $\beta 2 \mathrm{~N}$ in $\beta 2 \mathrm{KO}$ were similar to WT BK $\mathrm{BK}_{\mathrm{i}}$ currents recorded during the day $(\beta 2 \mathrm{KO}+\beta 2 \mathrm{~N}$ at $+90 \mathrm{mV}: 40 \pm 4 \mathrm{~ms}, n=20$, Fig. 6a; Supplementary Fig. $2 \mathrm{c}, \mathrm{d}$ ). Furthermore, $\beta 2 \mathrm{~N}$ decreased $\beta 2 \mathrm{KO}$ BK current levels back to levels comparable to WT neurons (Fig. 6b), without affecting the voltage dependence of BK current activation (Supplementary Fig. 5) or other voltage-activated outward currents (Supplementary Fig. 6). The restoration of daytime BK current levels by $\beta 2 \mathrm{~N}$ support the conclusion that inactivation is the critical function of the $\beta 2$ subunit that underlies its role in driving the diurnal variation in BK current properties in SCN.

Inactivation controls daytime membrane properties and firing. If inactivation is required for the diurnal difference in $\mathrm{BK}$ currents, then loss of the $\beta 2$ subunit or selective rescue of inactivation with $\beta 2 \mathrm{~N}$ should alter neuronal activity. To test this, we recorded spontaneous action potentials from WT and $\beta 2$ KO SCN neurons. WT neurons fire at higher frequencies during the day and decrease firing at night (Fig. 7a,b). Neurons with $\mathrm{BK}_{\mathrm{i}}$ currents fired faster than those with $\mathrm{BK}_{\mathrm{s}}$ currents regardless of time of day, suggesting that inactivation may play a role in setting firing rate. Furthermore, the relative difference in firing between $\mathrm{BK}_{\mathrm{i}}$ and $\mathrm{BK}_{\mathrm{s}}$ neurons during the day was as large as the net 

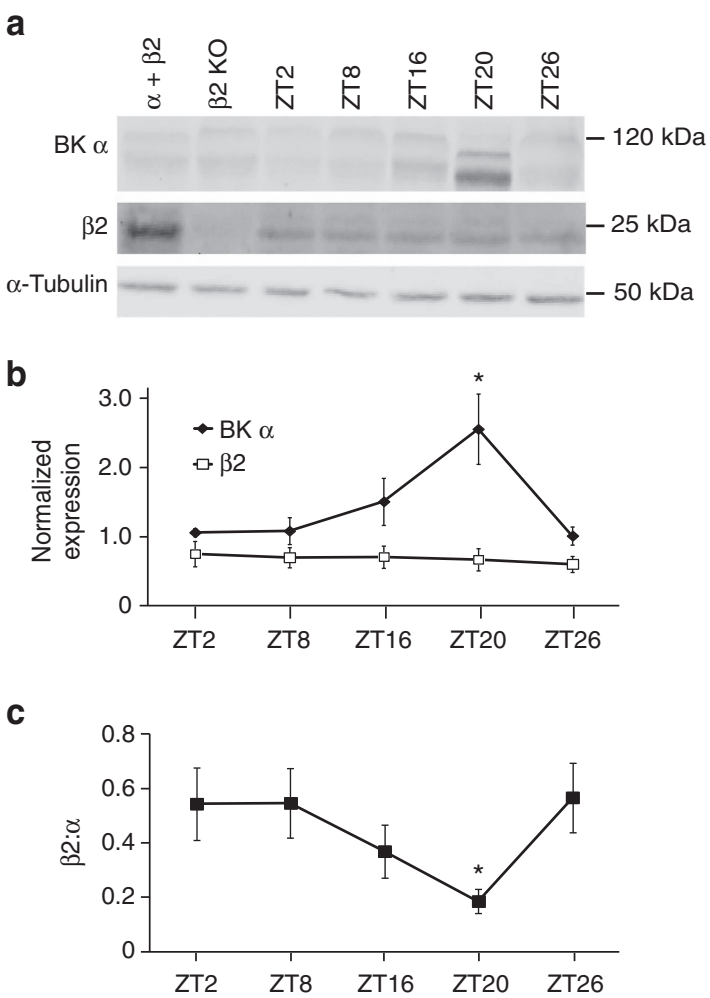

Figure 3 | Expression of BK $\alpha$ and $\mathbf{\beta 2}$ in WT SCNs. (a) BK channel complexes were immunoprecipitated with an anti-BK $\alpha$ subunit antibody from WT SCNs at the indicated time points. Western blot analysis was performed for BK $\alpha$ (top panel), $\beta 2$ (middle) or $\alpha$-tubulin (bottom). $\alpha$-Tubulin westerns (loading control) were obtained by running an equivalent volume of supernatant as was used for the immunoprecipitation. Protein was also harvested from $\alpha+\beta 2$ subunits co-expressed in HEK293 cells (positive control) or from $\beta 2 \mathrm{KO} \mathrm{SCNs}$ (negative control). ZT, zeitgeber time. Images have been cropped for presentation. Full size images are presented in Supplementary Fig. 3. (b) BK $\alpha$ and $\beta 2$ band intensities normalized to $\alpha$-tubulin. $\alpha$ expression increases at night, while $\beta 2$ expression does not change. Data are the average from four independent timed SCN collections (four SCNs at each timepoint). (c) Ratio of $\beta 2: \alpha$ expression in WT SCNs across the circadian cycle. All values are mean \pm s.e.m. ${ }^{\star} P<0.05$, Bonferroni post hoc.

diurnal difference in firing across the SCN (Fig. 7b), suggesting $\mathrm{BK}$ inactivation may be fundamental to the diurnal regulation of firing rate. Consistent with this, $\beta 2 \mathrm{KO}$ neurons did not exhibit a day-night difference in firing rate, stemming from a marked reduction in daytime firing (Fig. $7 \mathrm{~b}, \mathrm{c})$. To verify that the reduced firing, which was correlated with larger $\mathrm{BK}$ currents in $\beta 2 \mathrm{KO}$ neurons (Fig. 6b), was due to $\mathrm{BK}$, the $\mathrm{BK}$ channel blocker paxilline was applied. The reduced firing in $\beta 2 \mathrm{KO}$ neurons was reversed by paxilline (Fig. 7b), demonstrating that BK channels were responsible for the altered excitability in $\beta 2 \mathrm{KO}$ neurons.

These results corroborate the reduced peak firing observed in SCN circuit recordings (Fig. 1e). In addition, these data may also explain why loss of $\mathrm{BK}$ currents during the day has apparently little effect on WT firing rates ${ }^{24,43}$. A detectable change in firing after BK block in WT neurons may be precluded under standard daytime conditions when two-thirds of SCN neurons undergo BK inactivation. Following this idea, application of $\beta 2 \mathrm{~N}$, but not $\beta 2 \mathrm{~N}^{\Delta \mathrm{FIW}}$, increased daytime $\beta 2 \mathrm{KO}$ firing rates back to WT levels (Fig. $7 \mathrm{~b}, \mathrm{~d}$ ). $\beta 2 \mathrm{~N}$ had no further consequences on firing beyond that of blocking the BK current with paxilline (Fig. 7b), demonstrating that the restoration of firing with $\beta 2 \mathrm{~N}$ acts a
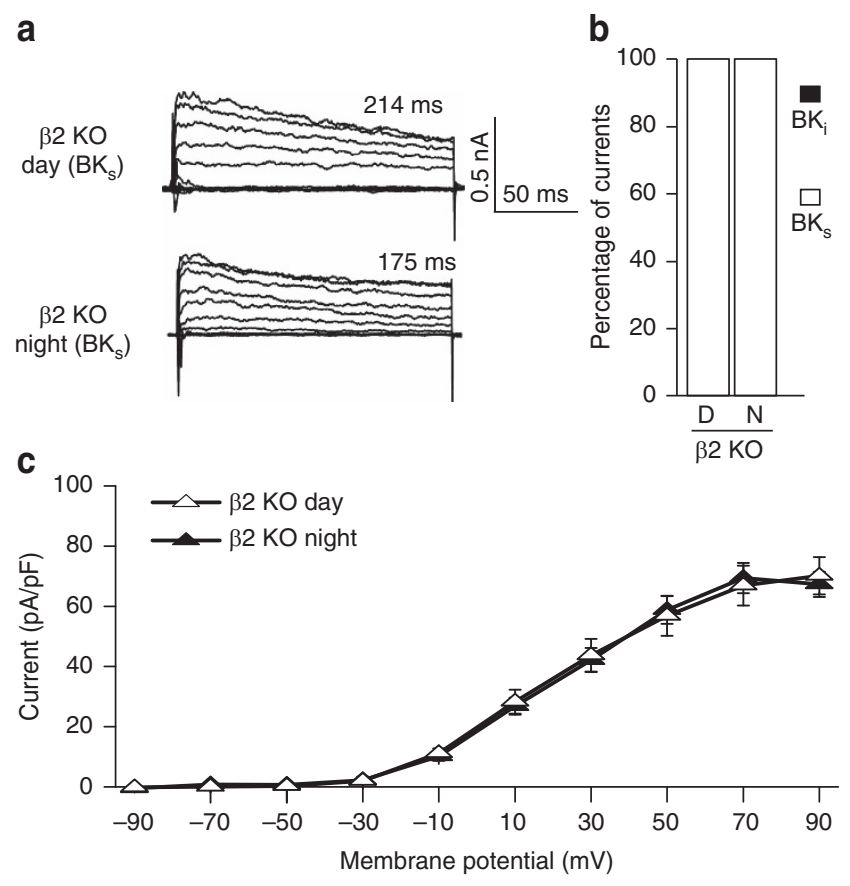

d

e
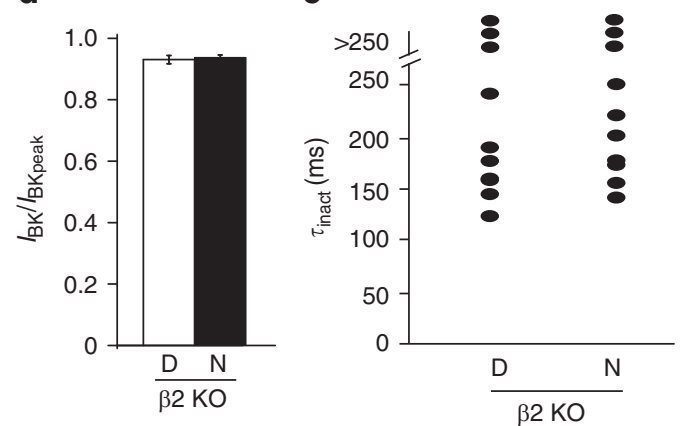

Figure 4 | The $\beta 2$ subunit is required for $B K_{i}$ current decay and the diurnal difference in BK current magnitude in SCN neurons.

(a) Representative BK current traces and macroscopic decay time constants $\left(\tau_{\text {inact }}\right)$ for $\mathrm{BK}_{\mathrm{s}}$ currents from $\beta 2 \mathrm{KO}$ neurons. Voltage protocol same as in Figure 2. (b) All currents are $\mathrm{BK}_{\mathrm{s}}$ from $\beta 2 \mathrm{KO} \mathrm{SCNs}$, day or night. (c) $\beta 2 \mathrm{KO}$ neurons do not show a day-night difference in BK current density. The $\beta 2 \mathrm{KO}$ daytime BK current magnitude is larger than WT, comparable to WT levels at night. (d) The fractional BK current (/ $30 \mathrm{~ms} /$ $I_{\text {peak }}$ ) was similar during the day compared with night in $\beta 2 \mathrm{KO}$ neurons. (e)

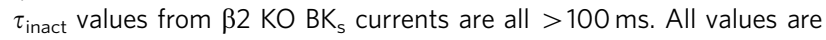
mean \pm s.e.m. $n$ values: $\beta 2 \mathrm{KO}$, day (20) and night (20).

selectively through $\mathrm{BK}$ channels. These results suggest that inactivation normally removes BK currents from influencing action potential firing rate during the day. Illustrating this in another way, $\beta 2 \mathrm{~N}$ could also aberrantly suppress BK current (Fig. 8a-c) and elevate firing if applied to WT neurons at night (Fig. 8d), underscoring the basis for the normal reduction in the number of $\mathrm{BK}_{\mathrm{i}}$ currents found in the SCN at night.

What membrane properties does $\mathrm{BK}$ inactivation regulate to facilitate daytime firing? A central node for circadian regulation of action potential frequency is the balance of subthreshold excitatory and inhibitory currents that bring the resting membrane potential closer to the firing threshold during the day, or further from threshold at night ${ }^{26,44}$. $\mathrm{K}^{+}$currents are proposed to be central regulators of the day-night difference in firing by regulating the daily transition between the daytime 
a

a

$0 \quad 10 \mu \mathrm{M}$
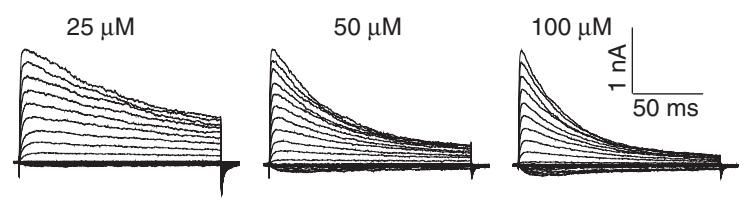

b

$50 \mu \mathrm{M}$

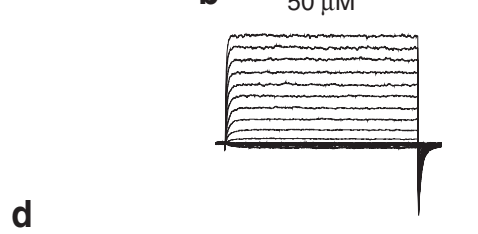

C

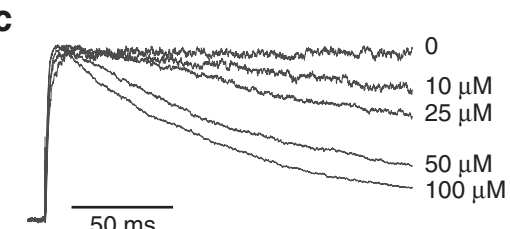

d

e

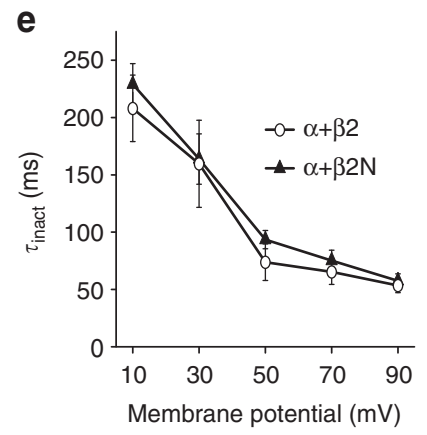

Figure 5 | The $\boldsymbol{\beta 2} \mathbf{N}$ terminus $(\boldsymbol{\beta 2} \mathbf{N})$ causes inactivation of BK currents from $\boldsymbol{\alpha}$-only channels expressed in heterologous cells. (a) Representative macroscopic current traces from HEK293 cells expressing BK SRKR channels, a daytime BK variant previously cloned from SCN (ref. 60). $\beta 2 \mathrm{~N}(0-100 \mu \mathrm{M}$ ), corresponding to the first 45 amino acids of the $\mathrm{N}$ terminus, was dissolved in recording solution and applied to the intracellular side via the patch pipette. (b) Representative currents in the presence of $50 \mu \mathrm{M} \beta 2 \mathrm{~N}^{\Delta \mathrm{FIW}}$, a peptide mutating the three residues required for inactivation ${ }^{42}$. There was no macroscopic current decay with $\beta 2 \mathrm{~N}^{\Delta \mathrm{FIW}}$. (c) Representative traces from the $+90 \mathrm{mV}$ voltage step at each concentration of $\beta 2 \mathrm{~N}$. The current peaks were scaled to illustrate the dose-dependent speeding of inactivation. (d) $\tau_{\text {inact }}$ values were calculated from the current elicited at $+90 \mathrm{mV}$ and plotted as a function of $\beta 2 \mathrm{~N}$ concentration. $\tau_{\text {inact }}$ was plotted for $\mathrm{BK}_{i}$ and $\beta 2 \mathrm{KO} S \mathrm{SCN}$ BK currents for cross-comparison. (e) $\tau_{\text {inact }}$ versus voltage for $\mathrm{BK}$ channels coexpressed the $\beta 2$ subunit, or with $50 \mu \mathrm{M} \beta 2 \mathrm{~N}$. There is no difference in the voltage dependence of activation using the isolated $\beta 2 \mathrm{~N}$ compared with the intact $\beta 2$ subunit. All values are mean \pm s.e.m. For currents from HEK293 patches, $n=4$ at each concentration of $\beta 2 \mathrm{~N}$ or $\beta 2 \mathrm{~N}^{\Delta \mathrm{FIW}}$ peptide. For currents from daytime $\mathrm{SCN}$ neurons, $\mathrm{BK}_{\mathrm{i}}(n=18)$ and $\beta 2 \mathrm{KO}+\beta 2 \mathrm{~N}(n=10)$.

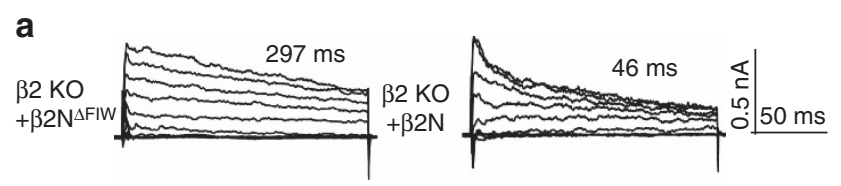

b

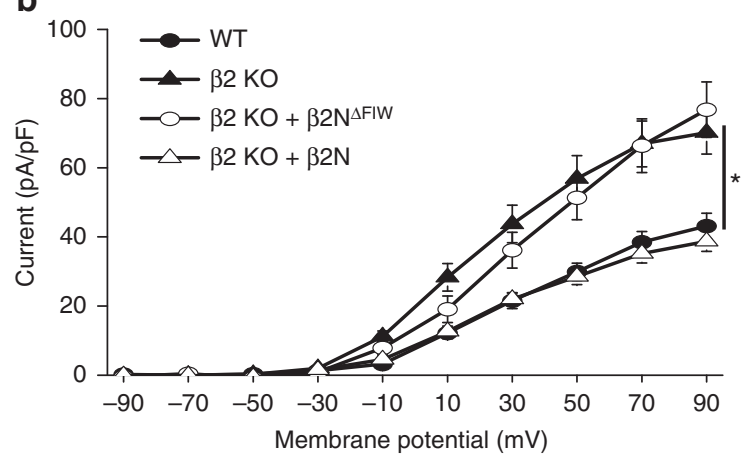

Figure 6 | $\beta 2 \mathrm{~N}$ rescues inactivation and restores daytime BK current levels in $\mathbf{\beta 2}$ KO SCN neurons. (a) Representative traces showing rescue of the macroscopic BK current decay in $\beta 2 \mathrm{KO}$ neurons with $50 \mu \mathrm{M} \beta 2 \mathrm{~N}$ applied intracellularly. $50 \mu \mathrm{M} \beta 2 \mathrm{~N}^{\Delta \mathrm{FIW}}$ did not rescue the current decay. Voltage protocol same as in Fig. 2. (b)Application of $\beta 2 \mathrm{~N}$ reduced the BK current density in $\beta 2 \mathrm{KO}$ neurons to levels comparable to WT. $\beta 2 \mathrm{~N}^{\Delta \mathrm{FIW}}$ had no effect on BK current levels. WT and $\beta 2 \mathrm{KO}$ I-Vs re-plotted from Figs 2 and 4 for cross-comparison. $n$ values: WT (27); $\beta 2 \mathrm{KO}(20) ; \beta 2 \mathrm{KO}+\beta 2 \mathrm{~N}^{\Delta \mathrm{FIW}}(18) ; \beta 2$ $\mathrm{KO}+\beta 2 \mathrm{~N}(20)$. All values are mean \pm s.e.m. ${ }^{\star} P<0.05$, Bonferroni post hoc. 'upstate' and night time 'downstate ${ }^{44-47}$. These states are designated by differences in the subthreshold membrane properties, with the most attention focused on the increase in night time $\mathrm{K}^{+}$current associated with hyperpolarization of the membrane and lower input resistance ${ }^{44,45,47,48}$. BK channels comprise a component of this upregulated $\mathrm{K}^{+}$current, and loss or block of $\mathrm{BK}$ currents causes depolarization of the baseline membrane potential and hyperactive firing at night ${ }^{23-25}$. However, block of BK currents also depolarizes the membrane potential during the day ${ }^{43,47,49}$, suggesting a role for BK channels in daytime excitability not previously explored. Thus to determine whether $\mathrm{BK}$ inactivation makes a converse contribution to the daytime reduction in $\mathrm{K}^{+}$current that defines the 'upstate' that is required for higher frequency firing, we compared membrane parameters from neurons during the day and night, and with loss of $\beta 2$ or restoration of inactivation.

First, we found that $\mathrm{BK}_{\mathrm{i}}$ neurons had inter-spike membrane potentials ranging from -36 to $-51 \mathrm{mV}$, while $\mathrm{BK}_{\mathrm{s}}$ neurons ranged from -47 to $-54 \mathrm{mV}$ during the day. Loss of inactivation in $\beta 2 \mathrm{KO}$ neurons resulted in hyperpolarization of the inter-spike membrane potential compared with $\mathrm{WT} \mathrm{BK}_{\mathrm{i}}$ neurons $(-57 \pm 1.4 \mathrm{mV}$ versus $-50 \pm 1.1 \mathrm{mV}$, respectively; $P<0.001$, $t$-test). This shift could be rescued by $\beta 2 \mathrm{~N}$ $(-52 \pm 0.8 \mathrm{mV})$, but not $\beta 2 \mathrm{~N}^{\Delta \mathrm{FIW}}(-56 \pm 1.5 \mathrm{mV}$; one-way ANOVA, $P=0.04$; Bonferroni post hoc, $P<0.05$ for $\mathrm{BK}_{\mathrm{i}}$ versus $\beta 2 \mathrm{KO}$ or $\left.\beta 2 \mathrm{~N}^{\Delta \mathrm{FIW}}\right)$, suggesting that $\mathrm{BK}$ channel inactivation affects membrane potential during the inter-spike interval. To further address this, resting membrane potential $\left(V_{\mathrm{m}}\right)$ and input 

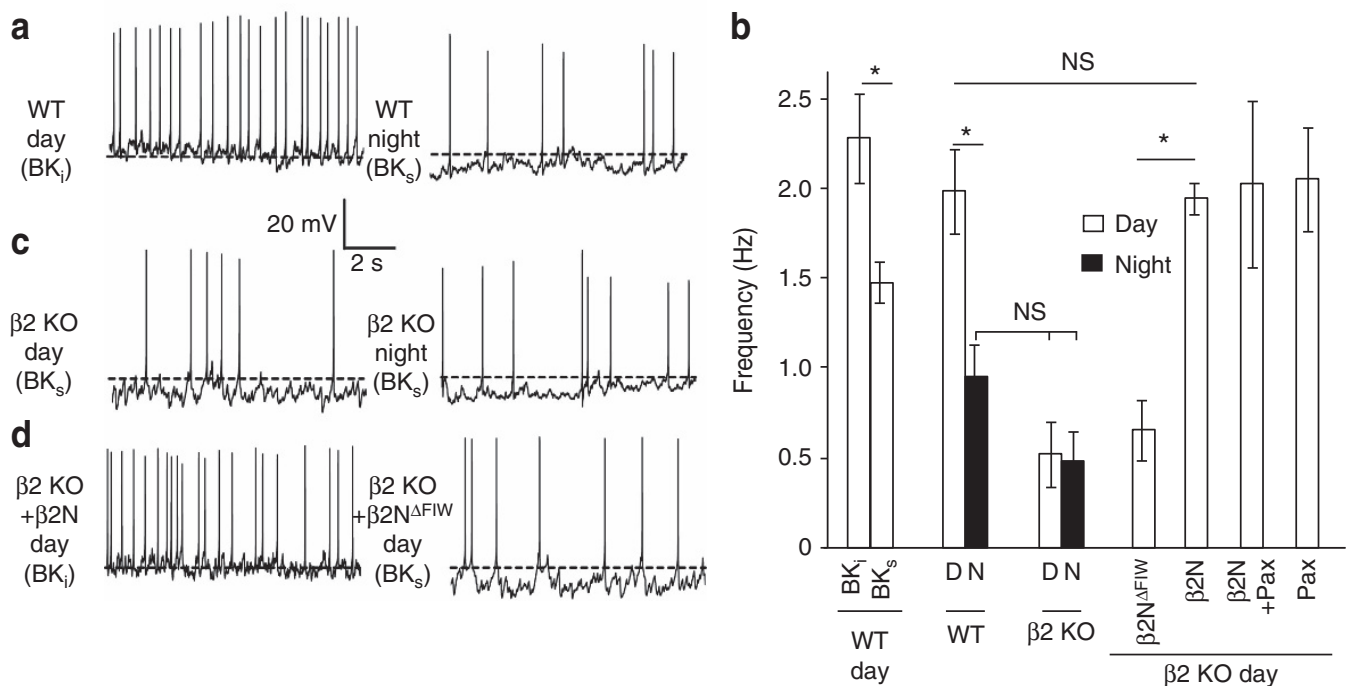

Figure 7 | Loss of $\beta 2$ eliminates the diurnal difference in firing rate, and rescue of inactivation with $\beta 2 \mathrm{~N}$ restores daytime firing rates in SCN neurons. (a) Spontaneous action potential activity from representative day $\left(\mathrm{BK}_{\mathrm{i}}\right)$ and night $\left(\mathrm{BK}_{\mathrm{s}}\right)$ WT neurons. Dotted line $(\mathbf{a}, \mathbf{c}, \mathbf{d})$ denotes $-50 \mathrm{mV}$. (b) In WT SCNs, $\mathrm{BK}_{\mathrm{i}}$ neurons fired at higher frequencies than $\mathrm{BK}_{\mathrm{s}}$, similar to the average day-night difference in firing. $\beta 2 \mathrm{KO}$ neurons did not exhibit a diurnal difference in frequency, and during the day, fired at levels similar to WT night. Application of $\beta 2 \mathrm{~N}^{\Delta \mathrm{FIW}}$ to daytime $\beta 2 \mathrm{KO}$ neurons had no effect on frequency, but $\beta 2 \mathrm{~N}$ increased firing rate to WT levels. (c) Day $\left(B_{s}\right)$ and night $\left(B K_{s}\right) \beta 2$ KO neurons. (d) Day $\beta 2 \mathrm{KO}$ neurons with $50 \mu \mathrm{M} \beta 2 \mathrm{~N}\left(\mathrm{BK}_{\mathrm{i}}\right)$ or $50 \mu \mathrm{M} \beta 2 \mathrm{~N}^{\Delta \mathrm{FIW}}\left(\mathrm{BK} \mathrm{s}_{\mathrm{s}}\right.$ ). All values are mean \pm s.e.m. $n$ values: $W T: B K_{i}(17), B K_{s}(10)$, day (17), night (20); $\beta 2$ KO: day (19), night (19), $\beta 2 \mathrm{~N}^{\Delta \mathrm{FIW}}(20), \beta 2 \mathrm{~N}(19), \beta 2 \mathrm{~N} / \mathrm{pax}(8)$, and $\operatorname{pax}(8)$.
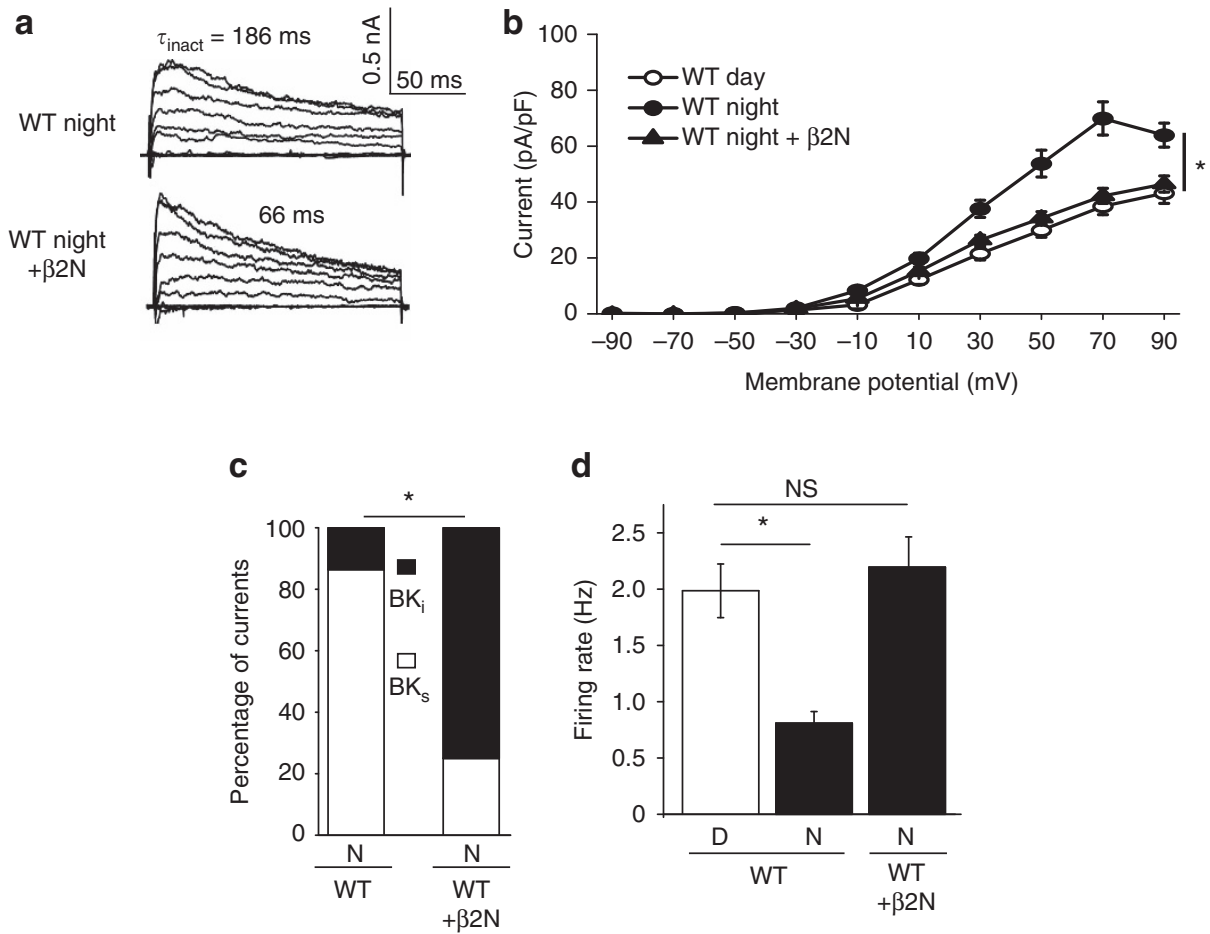

Figure 8 I $\mathbf{3 2 N}$ can confer inactivation to WT neurons at night. (a) Representative macroscopic traces from WT neurons at night showing a typical BK current, and a $\mathrm{BK}_{i}$ current resulting from application of $50 \mu \mathrm{M} \beta 2 \mathrm{~N}$. Voltage protocol same as in Fig. 2. (b) $\beta 2 \mathrm{~N}$ reduced the night time current density in WT neurons to daytime levels. WT day and night data re-plotted from Figs 2 and 4 for cross-comparison. (c) The proportion of BK $\mathrm{K}_{i}$ currents increased significantly with $\beta 2 \mathrm{~N}(P=0.0001$, Fisher's exact test). (d) $\beta 2 \mathrm{~N}$ increased night time firing to daytime levels. WT day and night data re-plotted from Fig. 7 for cross-comparison. All values are mean \pm s.e.m. $n$ Values: $W T$, day (27); WT, night (22); and $W T+\beta 2 N$, night $(20)$. ${ }^{\star} P<0.05$, Bonferroni post hoc.

resistance $\left(R_{\mathrm{i}}\right)$ recordings were made in the presence of tetrodotoxin to block action potential activity. Daytime neurons had more positive $V_{\mathrm{m}}$ and higher $R_{\mathrm{i}}$, while night time neurons had more hyperpolarized $V_{\mathrm{m}}$ and lower $\mathrm{R}_{\mathrm{i}}$ (Fig. 9a,b), consistent with previous studies ${ }^{45,48}$. The average daytime membrane potential $(-48 \pm 0.8 \mathrm{mV})$ was dominated by the increased number of $\mathrm{BK}_{\mathrm{i}}$ neurons, which had more positive $V_{\mathrm{m}}$ $(-47 \pm 0.4 \mathrm{mV})$ and higher $R_{\mathrm{i}}(1.4 \pm 0.06 \mathrm{G} \Omega)$ than $\mathrm{BK}_{\mathrm{s}}$ neurons 
a

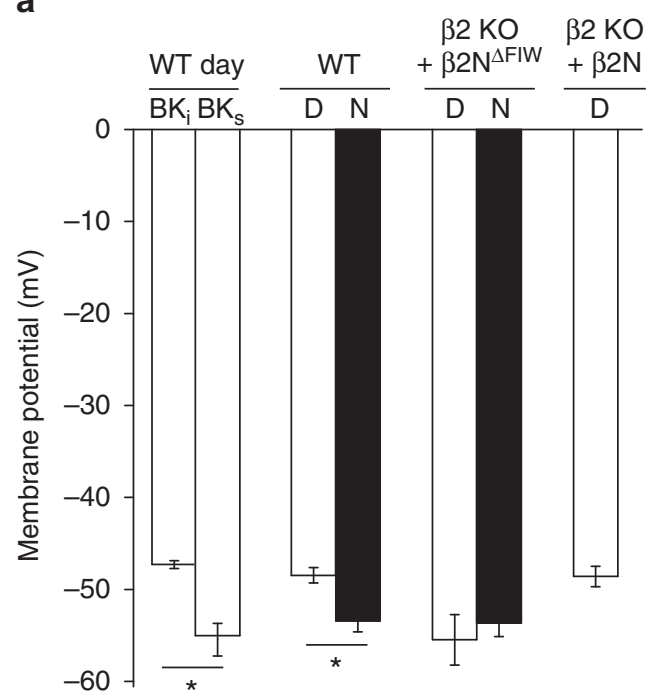

b

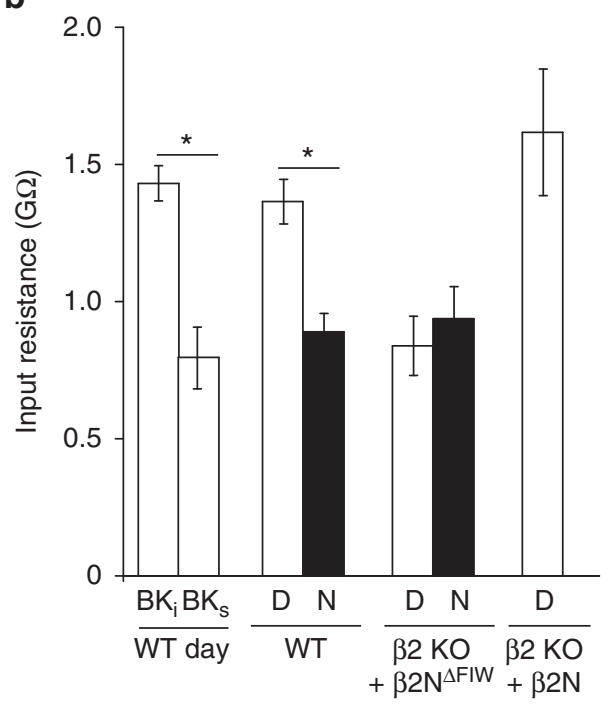

Figure 9 | BK current inactivation alters membrane potentials and input resistance in SCN neurons. (a,b) Resting membrane potentials (a) and input resistance (b) measured in $1 \mu \mathrm{M}$ tetrodotoxin to block action potentials. Conditions with inactivation $\left(\mathrm{BK}_{\mathrm{i}}, \beta 2 \mathrm{KO} / \beta 2 \mathrm{~N}\right.$, and WT day overall) had more depolarized $V_{\mathrm{m}}$ and higher $R_{\mathrm{i}}$ than $\mathrm{BK}_{\mathrm{s}}$, night, or $\beta 2 \mathrm{KO}$. All values are mean \pm s.e.m. $n$ values: WT: day/BK $/ K_{i}(9)$, day/BK ${ }_{s}(5)$, day (25), night (18) and $\beta 2 \mathrm{KO}: \beta 2 \mathrm{~N}^{\Delta \mathrm{FIW}} /$ day (11), $\beta 2 \mathrm{~N}^{\Delta \mathrm{FIW}} /$ night (10), $\beta 2 \mathrm{~N} /$ day (12). ${ }^{\star} P<0.05$, Bonferroni post hoc.

( $-55 \pm 1.7 \mathrm{mV}$ and $0.7 \pm 0.1 \mathrm{G} \Omega$, respectively; Fig. 9a,b). This suggests less current would be required to elicit an action potential in $\mathrm{BK}_{\mathrm{i}}$ compared with $\mathrm{BK}_{\mathrm{s}}$ neurons.

If inactivation of $\mathrm{BK}$ currents is involved in the changes in $V_{\mathrm{m}}$ and $R_{\mathrm{i}}$ between $\mathrm{BK}_{\mathrm{i}}$ and $\mathrm{BK}$ seurons, then loss of $\beta 2$ should also alter these parameters. We found that the $V_{\mathrm{m}}$ and $R_{\mathrm{i}}$ of daytime $\beta 2 \mathrm{KO}$ neurons was more similar to WT neurons at night (Fig. 9a,b), suggesting $\beta 2$ is required for the daytime 'upstate'. Furthermore, $\beta 2 \mathrm{~N}$ restored the $V_{\mathrm{m}}$ and $R_{\mathrm{i}}$ values in $\beta 2 \mathrm{KO}$ neurons back to values comparable to WT, establishing the sufficiency for inactivation of the $\mathrm{BK}$ current to convert the daytime membranes to pro-excitable. Although $\beta 2$ can also affect excitability by shifting the voltage dependence of activation and slowing activation and deactivation gating ${ }^{18,21}$ (Supplementary
Discussion), the data presented here affirm that these functions are not directly involved in the diurnal regulation of membrane properties that control firing frequency in SCN.

The effect of inactivation on resting membrane potential and input resistance predicts that inactivation of $\mathrm{BK}$ currents would occur during the inter-spike interval. To demonstrate this, we recorded BK currents from traditional voltage step commands across the subthreshold range of membrane potentials, as well as BK currents evoked by SCN action potential commands (Fig. 10; Supplementary Fig. 7). $\mathrm{BK}_{\mathrm{s}}$ current-voltage relationships showed that daytime $\mathrm{BK}$ current activated at voltages more positive than $-60 \mathrm{mV}$ (Fig. 10a,b). There was 5-12 pA of current evoked in the subthreshold range, between the average resting and threshold potentials of SCN neurons $(-55$ to $-40 \mathrm{mV})$. Night time subthreshold $\mathrm{BK}$ currents $\left(\mathrm{BK}_{\mathrm{s}}\right)$ were also larger than the net daytime BK current (Supplementary Fig. 7a). In contrast, daytime $\mathrm{BK}_{\mathrm{i}}$ neurons had significantly reduced current in the subthreshold voltage range (Fig. 10a,b). Consistent with data obtained from voltage steps, using daytime SCN action potential commands delivered at the normal daytime firing frequency, the amount of BK current activated during the inter-spike interval was negligible in $\mathrm{BK}_{\mathrm{i}}$ neurons compared with $\mathrm{BK}_{\mathrm{s}}$ neurons (Fig. 10c,d) or at night (Supplementary Fig. $7 \mathrm{~b}, \mathrm{c}$ ). BK currents evoked at the peak of the action potential command were also reduced in $\mathrm{BK}_{\mathrm{i}}$ neurons compared with $\mathrm{BK}_{\mathrm{s}}$ neurons during the day (Fig. 10e) or at night (Supplementary Fig. 7d). In parallel experiments using daytime action potential commands applied to channel complexes expressed in HEK293 cells (Supplementary Fig. 8a-j), the subthreshold current was reduced in patches containing $B K / \beta 2$ or $B K / \beta 2 N$ compared with $\alpha$-only channels (Supplementary Fig. $8 \mathrm{f}-\mathrm{i}$ ).

To further demonstrate that inactivation could affect BK current levels at these subthreshold voltages in SCNs, $\beta 2 \mathrm{KO}$ neurons were held at a conditioning voltage for $100 \mathrm{~ms}$, corresponding to the shortest inter-spike intervals encountered in spontaneously firing SCN neurons, followed by a step to $+90 \mathrm{mV}$ to elicit a maximal BK current response (Fig. 10f). Inactivation delivered via $\beta 2 \mathrm{~N}$ reduced the maximal $\mathrm{BK}$ current elicited following conditioning voltages in the subthreshold range, compared with $\beta 2 \mathrm{KO}$ cells treated with $\beta 2 \mathrm{~N}^{\Delta \text { FIW }}$ (Fig. 10f,g). Further depolarization of the conditioning potential resulted in progressive current reduction that was dependent on the presence of inactivation (Fig. 10f,g). Similar results were obtained with BK channels expressed in heterologous cells with the intact $\beta 2$ subunit or with $\beta 2 \mathrm{~N}$ (Supplementary Fig. 8a,b). These results suggest that inactivation can reduce BK current under conditions similar to the native inter-spike interval and provide a basis for understanding the effect of $\mathrm{BK}$ inactivation on the resting membrane potential and input resistance.

The data further propose that because some BK current would be inactivated before an action potential, the current evoked by an action potential would be reduced. This would be expected to occur during the day in $\mathrm{BK}_{\mathrm{i}}$ neurons. To test this, we quantified the peak BK current evoked by daytime action potentials applied to SCN neurons (Fig. $10 \mathrm{c}, \mathrm{e}$ ) and to $\mathrm{BK} / \beta 2$ or $\mathrm{BK} / \beta 2 \mathrm{~N}$ channels expressed in heterologous cells (Supplementary Fig. 8e-h,j). The action potential-evoked $\mathrm{BK}$ current was reduced in $\mathrm{BK}_{\mathrm{i}}$ versus $\mathrm{BK}_{\mathrm{s}}$ neurons (Fig. 10e) and in $\mathrm{BK} / \beta 2$ or $\mathrm{BK} / \beta 2 \mathrm{~N}$ conditions in HEK293 cells compared with $\alpha$-only channels (Supplementary Fig. 8j). Taken together, these data support a model where BK current inactivation is necessary during the day to regulate the membrane parameters that initiate firing (membrane potential and input resistance), while concurrently reducing the BK current activated during an action potential. This idea further predicts there would be little difference in the action potential waveforms of $\mathrm{BK}_{\mathrm{i}}$ versus $\mathrm{BK}_{\mathrm{s}}$ neurons, an observation confirmed by analysis of daytime action potential parameters (Supplementary Fig. 9). 
a

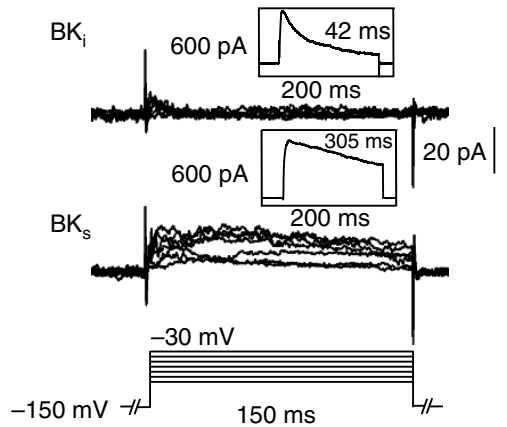

C

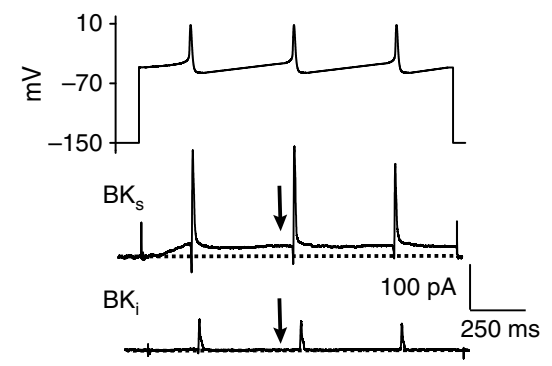

f

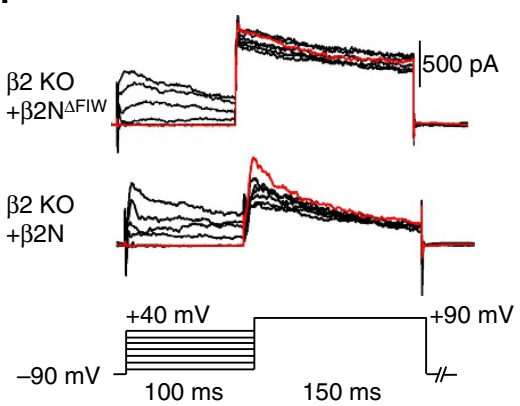

b

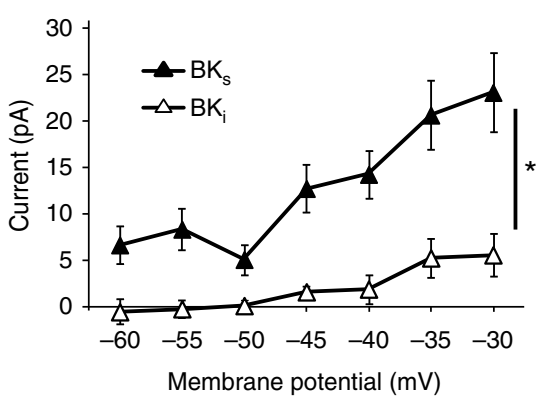

d
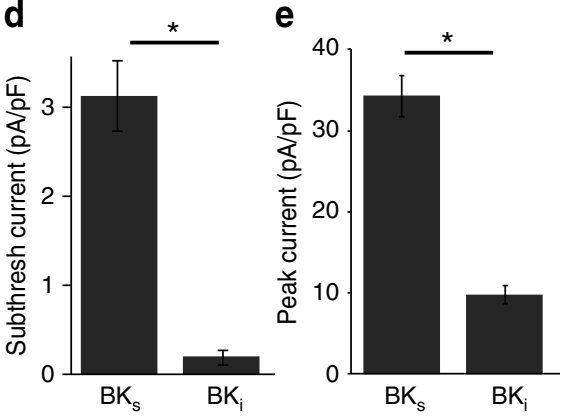

g

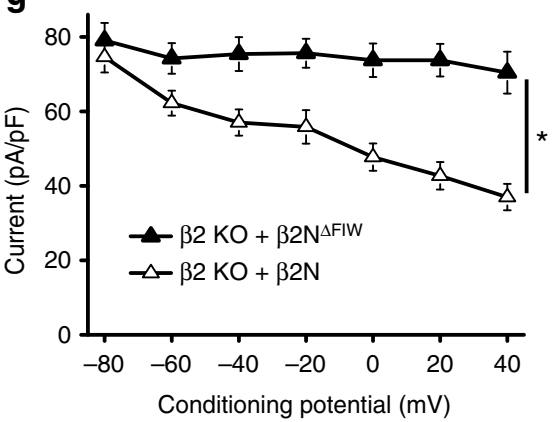

Figure 10 | Steady-state and action potential-evoked $\mathbf{B K}_{\mathbf{i}}$ currents are reduced compared to $\mathbf{B K}_{\mathbf{s}}$ in $\mathbf{S C N}$ neurons. (a) Representative macroscopic BK current traces from daytime WT neurons in response to a subthreshold voltage protocol ( -60 to $-30 \mathrm{mV}$ in $5 \mathrm{mV}$ steps). BK currents were identified as $B K_{i}$ or $B K_{s}$ from a maximally activating step to $+90 \mathrm{mV}$ (as in Fig. 2). (b) Current-voltage relationship showing significantly more activation of $B K_{s}$ current above $-60 \mathrm{mV}$ compared to $\mathrm{BK}_{\mathrm{i}}$. (c) Pre-recorded action potential commands (top) were used to elicit $B K$ currents from $B K_{i}$ or $B K_{s} n e u r o n s$. From a holding potential of $-150 \mathrm{mV}$ to remove inactivation completely, cells were stepped to the inter-spike potential $(-48 \mathrm{mV})$ with a sequence of three action potential commands as depicted (Peak, $8 \mathrm{mV} ; t_{1 / 2}, 5.5 \mathrm{~ms}$; and AHP/antipeak, $-54 \mathrm{mV}$ ). Arrow, the subthreshold current level in $\mathbf{d}$ was taken just before the second action potential command. (d,e) Average subthreshold BK current density from the inter-spike interval (d) or at the peak of the action potential (e). $n$ values for (a-d): WT: day/BK $(9)$, day/BK $(5)$. (f) BK current as a function of conditioning potential in SCN neurons. Representative macroscopic BK current traces from $\beta 2 \mathrm{KO}$ neurons during the day with $50 \mu \mathrm{M} \beta 2 \mathrm{~N}^{\Delta \mathrm{FIW}}$ or $\beta 2 \mathrm{~N}$. Neurons were held at $-90 \mathrm{mV}$ for $150 \mathrm{~ms}$ to remove inactivation, and then stepped to a conditioning potential for $100 \mathrm{~ms}$ to allow channels to transition into the inactivated state, followed by a maximally activating step to $+90 \mathrm{mV}$. (g) The peak BK current elicited from the $+90 \mathrm{mV}$ step was plotted as a function of the conditioning potential. $\beta 2 \mathrm{~N}$ causes a reduction in current, but no reduction is observed in the absence of inactivation with $\beta 2 \mathrm{~N}^{\Delta \mathrm{FIW}} . n=5$ for each condition. All values are mean \pm s.e.m. ${ }^{\star} P<0.05$,

Bonferroni post hoc.

$\beta 2$-mediated inactivation thus comprises an essential switch that shifts membrane properties between day and night states by inhibiting or permitting subthreshold BK current activation.

\section{Discussion}

The circadian patterning of neuronal excitability is an evolutionarily conserved feature of time-encoding circuits ${ }^{26,50}$. In SCN neurons, which fire relatively slow, spontaneous action potentials characteristic of pacemaker neurons ${ }^{49}$, the balance of currents during the inter-spike interval is proposed to be the predominant regulator of firing rate and a major node for circadian regulation $26,44,45,48$. Correlated with the increased number of $\mathrm{BK}_{\mathrm{i}}$ currents in SCN during the day, most neurons have a more depolarized resting membrane potential that is closer to threshold ( $\Delta V \sim 7 \mathrm{mV}$, from our data), increasing the likelihood of firing an action potential. At night, and correlated with the shift to $\mathrm{BK}_{\mathrm{s}}$ currents, the hyperpolarizing shift places the membrane potential further from threshold $(\Delta V \sim 12 \mathrm{mV})$, reducing excitability. The amount of current required to produce this shift is small ( $\sim 5 \mathrm{pA})$, owing to the high input resistance of SCN neurons, and the balance can be tipped either by altering the 
excitatory currents that bring the membrane potential closer threshold, or the inhibitory currents that take the membrane away from threshold. Recently, the identity of the channel underlying the persistent $\mathrm{Na}^{+}$current that provides the depolarizing drive on excitability was identified as the leak $\mathrm{Na}^{+}$channel NALCN ${ }^{51}$. However, the identity of the $\mathrm{K}^{+}$ channels counterbalancing the excitatory current has been uncertain, particularly during the day when SCN neurons undergo a dramatic reduction in $\mathrm{K}^{+}$current $^{44,45,49}$. Although abrogation of one type of $\mathrm{K}^{+}$current during the day, the fast delayed rectifier, has also been shown to decrease firing frequency, the reduced firing stems from slower action potential repolarization and not from depolarization of the resting membrane potential ${ }^{52}$. Consequently, a direct test of whether the daily shift in submembrane $\mathrm{K}^{+}$currents is required to drive the day-night difference in action potential firing has been elusive.

In this study we provide surprising evidence linking alterations in BK current with the daytime increase in input resistance, depolarization of the membrane and higher frequency firing. BK currents were previously thought to mainly play a night time-restricted role as part of the surge of $\mathrm{K}^{+}$current that results in the firing nadir ${ }^{24,44,45}$. The less significant role for BK channels in setting daytime frequency was attributed to a decrease in expression of the pore-forming $\alpha$ subunit. However, in the $\beta 2$ $\mathrm{KO}$, the intact circadian pattern of $\alpha$ expression was insufficient to support normal diurnal variation in $\mathrm{BK}$ current or SCN firing, suggesting there must be other mechanisms that limit BK channels from influencing daytime membrane properties. We demonstrate that inactivation, achieved through $\beta 2$-dependent regulation of $\mathrm{BK}$ channel biophysical properties between day and night, is the central mechanism that changes the influence of BK channels on membrane properties between day and night. Inactivation facilitates the daytime membrane 'upstate' by reducing the $\mathrm{BK}$ current between spikes that would normally inhibit action potential initiation. Inactivation also reduces action potential-evoked current, and the sum of these two mechanisms effectively removes $\mathrm{BK}$ channels from influencing daytime excitability. In $\beta 2 \mathrm{KO}$ SCN neurons, the absence of $\mathrm{BK}$ inactivation during the day results in passive membrane properties that look much like night time membranes. Correspondingly, with $\beta 2 \mathrm{~N}$, restoration of inactivation is both necessary and sufficient to recapitulate the $V_{\mathrm{m}}$ and $R_{\mathrm{i}}$ properties that define the daytime membrane state. Importantly, the identification of this biophysical mechanism to functionally reduce a $\mathrm{K}^{+}$current suggests that the balance of excitatory and inhibitory currents is more actively regulated during the day than previously thought ${ }^{45}$. Moreover, the disruption of circadian behaviour in the absence of the $\beta 2$ subunit underscores the importance of both $\mathrm{BK}$ inactivation, and the diurnal variation in SCN submembrane properties, in the regulation of circadian rhythmicity. As such, $\mathrm{BK}_{\mathrm{i}}$ currents comprise an electrophysiological signature for a previously undetected class of essential oscillator in SCN that makes a vital contribution to circadian behaviour. Even so, the residual rhythmicity in $\beta 2 \mathrm{KOs}$ hints that neuronal heterogeneity contributes redundant mechanisms to circadian behavioural rhythmicity (Supplementary Discussion), and it remains to be determined how SCN neurons without inactivating BK channels balance their daytime currents to control firing, and what separable contribution these cells make to circadian behaviour.

Given that the BK current must be functionally reduced by inactivation, what is the relevance of the daytime clock-linked decrease in $\mathrm{BK} \alpha$ subunit expression ${ }^{24,30}$ ? Such differences in ion channel expression across the circadian cycle are broadly proposed to be central to rhythmicity ${ }^{26}$, but an explicit mechanism that translates channel expression differences into action potential activity has not been established. In this study, we showed $\beta 2$ KO SCNs retained the normal day-night difference in BK $\alpha$ expression, yet still displayed disrupted BK steady-state current levels and action potential firing, demonstrating that circadian differences in the $\mathrm{BK} \alpha$ subunit alone are insufficient to drive rhythmicity. Our data instead suggest a model in which the clock-linked changes in $\alpha$ expression control the ratio of $\beta 2: \alpha$. Since expression of $\beta 2$ does not vary appreciably between day and night, the higher daytime $\beta 2: \alpha$ ratio facilitates inactivation in the majority of SCN neurons, permitting transition to the 'upstate'. At night, inactivation is overcome by increased $\alpha$ subunit expression, reducing the $\beta 2: \alpha$ ratio and facilitating the 'downstate'. In support of this hypothesis, aberrantly increasing daytime BK expression reduces inactivation in the SCN (Supplementary Discussion).

The data in SCN also provide a framework for understanding the physiological relevance of $\mathrm{BK}$ channel inactivation, which is too slow to produce substantial BK current decay during a typical central nervous system action potential ${ }^{53}$. In other neurons where higher frequency firing prevails, $\mathrm{BK}$ inactivation is thought to contribute to spike frequency adaptation, which also integrates over timeframes longer than a single action potential ${ }^{10,15,17}$. In SCN neurons, which exhibit depolarized membrane potentials and slow spontaneous firing, the results here suggest that enough channels undergo inactivation, or remain cumulatively inactivated, during the inter-spike interval (100-500 ms) to impact the input resistance and resting membrane potential. Given $\tau_{\text {inact }}$ values of $35-100 \mathrm{~ms}$ in $\mathrm{BK}_{\mathrm{i}}$ neurons, steady-state inactivation may be most relevant for setting the subthreshold $\mathrm{BK}$ current level during the inter-spike interval. SCN neurons have been shown to undergo subthreshold $\mathrm{Ca}^{2+}$ oscillations ${ }^{49,54}$, raising the possibility that $\mathrm{Ca}^{2}+$ influx independent of the action potential could contribute to $\mathrm{BK}$ channel activation, and consequently $\mathrm{BK}$ inactivation, during the inter-spike interval. In addition, the restoration of firing rate with $\beta 2 \mathrm{~N}$ reveals that inactivation mediated by the $\mathrm{N}$ terminus is sufficient for this role. $\mathrm{N}$-type inactivation typically occurs from the open state $\mathrm{e}^{7,8}$, but BK channels may also inactivate from closed states, affecting the amount of steady-state current ${ }^{55}$. Thus, the data in this study reveal a surprising twist to how cells use $\mathrm{BK}$ channels to regulate excitability. While BK channels perform diverse roles spanning excitable and non-excitable cells, in the CNS they are mostly known for regulating firing frequency by underlying the fast $\mathrm{AHP}^{56-58}$. Here we show in SCN, BK current activated outside of the action potential is potentially the major contributor to frequency regulation, making dynamic regulation of subthreshold BK current via inactivation a novel role for BK channels.

In summary, although the combination of $\mathrm{BK}_{\mathrm{i}}$ and $\mathrm{BK}_{\mathrm{s}}$ expressing cells has been observed within other tissues ${ }^{18}$, the significance for how these currents work together to set the ensemble properties underpinning physiological function is not yet apparent ${ }^{53}$. In SCN, the cycling between predominantly $\mathrm{BK}_{\mathrm{i}}$ currents during the day and predominantly $\mathrm{BK}_{\mathrm{s}}$ currents at night suggests that partnering with the $\beta 2$ subunit generates a hardwired mechanism linked to the intrinsic clock to dynamically disengage $\mathrm{BK}$ channels from regulating action potentials during one phase of the circadian cycle.

\section{Methods}

Mice. WT, $\beta 2 \mathrm{KO}$ (KCNMB2 $2^{-/-}$, gift from C. Lingle), $\beta 4 \mathrm{KO}\left(\mathrm{KCNMB}^{-/-}\right.$, gift from R. Brenner), and Kcnma1 ${ }^{-9}-$ mice were maintained on inbred C57BL/6 background and genotyped as previously described ${ }^{18,24,59}$. KCNMB2 floxed mice are available at The Jackson Laboratory (Stock no. 028416). Male and female mice were used ( 3 weeks to 5 months old) and were group housed separated by sex until experimental procedures as indicated. Day time points were collected from mice housed on a standard $12 \mathrm{~h}$ light, $12 \mathrm{~h}$ dark cycle $(12: 12 \mathrm{LD})$, and night time points 
were collected from mice housed on a reverse 12:12 LD cycle. All procedures involving mice were conducted in accordance with the University of Maryland School of Medicine Animal Care and Use Guidelines and approved by the IACUC Committee.

Organotypic slice culture. Brains were harvested from postnatal day 4 mice, and the hypothalamus was blocked and sectioned into $300 \mu \mathrm{m}$ coronal slices using a manual chopper (Stoelting, Wood Dale, IL, USA). Slices containing SCN nuclei were transferred onto Millicell filters (Millipore, Billerica, MA, USA) and cultured as interface explants in a $\mathrm{CO}_{2}$ incubator as described ${ }^{23}$. After 2 days, $20 \mu \mathrm{M}$ cytosine $\beta$-D-arabinofuranoside (ara-C, no. C6645; Sigma) was added to inhibit glial proliferation, and the media was changed every 3 days thereafter.

Multielectrode array recordings. After 7 days in culture, organotypic slices were cut from the surrounding membrane, inverted over the 64-electrode grid, and adhered to $0.1 \%$ polyethylenimine and collagen-treated P210A probes (Alpha MED Scientific, Osaka, Japan). Probes were placed on a MED CO2P connector headstage, sealed with a vacuum-greased coverslip, and maintained in a humidified $5 \% \mathrm{CO}_{2}$ incubator at $37^{\circ} \mathrm{C}$ for the duration of the recordings. Media changes were maintained every 3 days.

Signals from all electrodes on the probe were collected simultaneously with the 64-channel integrated amplifier (Alpha MED Scientific) and analysed as described previously ${ }^{23}$. Five-second data samples were collected every $5 \mathrm{~min}$ at $20 \mathrm{kHz}$ in Conductor v3.1f (Alpha MED Scientific) and filtered at $100 \mathrm{~Hz}$. Spontaneous extracellular action potentials from visually identified electrodes within the SCN were discriminated offline using threshold-based event counting. Circuit analysis was performed on each slice from three cycles of activity at all electrodes within the SCN. The multiunit spontaneous action potential activity from each electrode located within the SCN was classified as rhythmic or arrhythmic. For rhythmic recordings, a $2 \mathrm{~h}$ moving window average was applied to the raw data to calculate the daily firing rate peak, and the 'day' firing rate value was the average of activity for $2 \mathrm{~h}$ centred around the peak for each recording. Firing rate at the trough was calculated from $2 \mathrm{~h}$ of activity at the nadir between two peaks. Arrhythmic firing was calculated as the average firing across the whole cycle. Circadian amplitude was reported as the $\chi^{2}$ periodogram peak value (Clocklab; Actimetrics, Wilmette, IL, USA). $\tau$, the length of the circadian period of the neural activity rhythm, was determined as the highest peak above the $99 \%$ confidence interval of a $\chi^{2}$ periodogram.

Circadian behavioural rhythms. For locomotor rhythms, mice (3-5 months old) were housed individually in cages containing a running wheel for 10 days in LD and 16 days in constant darkness (DD). Activity was sampled every $10 \mathrm{~min}$ in ClockLab software (Actimetrics). Actograms were constructed by double-plotting consecutive days of activity over the recording period. Circadian period and amplitude were determined from 10 days of wheel running activity in DD in Clocklab software as described previously ${ }^{23,24}$. For re-entrainment experiments, after 7 days of stable entrainment, the LD cycle was phase advanced by $6 \mathrm{~h}$. The response was calculated as the number of days to stable re-entrainment. After keeping mice in DD for 8 days, phase shifts in response to light pulses were calculated as the number of hours between the activity onset regression fits before and after a 30-min light pulse delivered in early subjective night (CT16). Alpha was determined as the length of time an animal had consolidated activity using default settings with manual adjustment, with rho defined as the portion of the cycle outside of alpha. Bouts were calculated using default settings. Data was excluded from mice that failed to run on wheels for two consecutive days.

Western blots. A single block of hypothalamus containing the SCN $(\sim 2 \mathrm{~mm})$ was harvested at the indicated time points from mice (2- to 3-months old). Four micrograms solubilized protein from individual SCNs were loaded per lane. Densitometry of BK doublet band $\left(1 \mu \mathrm{g} \mathrm{ml}{ }^{-1} \mathrm{~L} 6 / 60\right.$ mouse monoclonal $\alpha$-BK antibody, Neuromab; University of California, Davis, CA, USA) to DM1 $\alpha$ anti-tubulin (1:75,000; Sigma T-9026) was performed as described previously ${ }^{23}$ and presented as a proportion of ZT20. Three independent circadian cycle tissue harvests were performed for each condition.

For immunoprecipitation, individual SCNs $(\sim 50 \mu \mathrm{g}$ of protein) were precleared by incubation with $20 \mu \mathrm{l}$ TrueBlot anti-rabbit Ig IP beads (Rockland Immunochemicals; Limerick, PA, USA) for $2 \mathrm{~h}$ at $4{ }^{\circ} \mathrm{C}$ (ref. 18). Protein samples were centrifuged at 13,200 r.p.m. for $30 \mathrm{~min}$ to precipitate the beads. Supernatant was collected and mixed with $1 \mu \mathrm{g}$ of anti-BK $\alpha$ subunit antibody (anti-KCa1.1, \#1184-1200, Alamone Labs; Jerusalem, Israel) and rotated for $2 \mathrm{~h}$ at $4{ }^{\circ} \mathrm{C} .50 \mu \mathrm{l}$ TrueBlot anti-rabbit Ig IP beads were added, and the mixture was rotated overnight at $4{ }^{\circ} \mathrm{C}$, centrifuged at 13,200 r.p.m. for $30 \mathrm{~min}$ to precipitate beads, and the supernatant was discarded. Beads were washed three times and resuspended in $20 \mu \mathrm{l}$ lysis buffer. Protein was run on a western blot as detailed previously and probed with $1 \mu \mathrm{g} \mathrm{ml}^{-1}$ anti-BK (L6/60; Antibodies Incorporated; Davis, CA, USA); $1: 75,000$ anti- $\alpha$-Tubulin (DM1a; Sigma; St Louis, MO, USA); or $1 \mu \mathrm{g} \mathrm{ml}^{-1}$ anti- $\beta 2$ (N53/32; Antibodies Incorporated; Davis, CA, USA). Full size images for western blots are presented in Supplementary Figs 3 and 4.
Acute SCN slice preparation. Mice were sacrificed in the light, at zeitgeber time (ZT) ZT0-2 (day time points) or ZT11-12 (night) from 3- to 6-week-old mice. Brains were rapidly removed and placed into ice-cold sucrose-substituted saline containing the following (in mM): $1.2 \mathrm{MgSO}_{4}, 26 \mathrm{NaHCO}_{3}, 1.25 \mathrm{Na}_{2} \mathrm{HPO}_{4}, 3.5$ $\mathrm{KCl}, 3.8 \mathrm{MgCl}_{2}, 10$ glucose and 200 sucrose. Coronal slices were cut at $300 \mu \mathrm{m}$ on a VT1000S vibratome (Leica Microsystems, Wetzlar, Germany) at $3-4^{\circ} \mathrm{C}$. Slices containing SCN were recovered for $1-3 \mathrm{~h}$ (day) and $17-19 \mathrm{~h}$ (night) at $25^{\circ} \mathrm{C}$ submerged in oxygenated artificial cerebrospinal fluid (in mM: $125 \mathrm{NaCl}, 1.2$ $\mathrm{MgSO}_{4}, 26 \mathrm{NaHCO}_{3}, 1.25 \mathrm{Na}_{2} \mathrm{HPO}_{4}, 3.5 \mathrm{KCl}, 2.5 \mathrm{CaCl}_{2}$ and 10 glucose).

Slices containing SCN were transferred to the recording chamber (RC26GLP/PM-1; Warner Instruments, Hamden, CT, USA) with gravity flow bath perfusion of $1-2 \mathrm{ml} \mathrm{min}^{-1}$ oxygenated artificial cerebrospinal fluid at $25^{\circ} \mathrm{C}$. Neurons were visualized with a Luca-R DL-604 EMCCD camera (Andor, Belfast, UK) under IR-DIC illumination on an FN1 upright microscope (Nikon, Melville, NY, USA). Recordings were made from cells in the centre of the SCN. Current- and voltage-clamp recordings were made with a Multiclamp 200B or 700B amplifier and pCLAMP v10 software (Molecular Devices, Sunnyvale, CA, USA). Data were acquired at a 20 or $100 \mathrm{kHz}$ sampling rate. Drugs were delivered to the bath by a computer-controlled pressurized perfusion system (ValveLink 8.2; Automate Scientific, Berkeley, CA, USA) at the concentrations indicated from 1,000 $\times$ stocks. Recording windows were at the peak (ZT4-8) and nadir (ZT17-21) of the circadian rhythm in spontaneous action potential firing, corresponding to the 'day' and 'night' time points, respectively. Although the action potential frequencies in this study were lower than our previous studies performed at $35^{\circ}$ (refs 22,23), the day-night difference in firing frequency in this study is preserved (Fig. 4a,c). All recordings were made with synaptic transmission intact, unless indicated (Tetrodotoxin was applied for all $V_{\mathrm{m}}$ and $R_{\mathrm{i}}$ measurements, as well as the subthreshold BK currents in Fig. 5). Reported N's are the number of neurons, and data for each condition was derived from 5 to 15 slices (1-6 neuronal recordings per slice).

SCN electrophysiology. SCN neurons were recorded in whole-cell configuration, first in current-clamp mode to collect action potential data, then switched into voltage clamp mode to obtain macroscopic voltage-activated outward $\mathrm{K}^{+}$currents. Electrodes (4-7 M $\Omega$ ) were filled with internal solution, in mM: $123 \mathrm{~K}$-methanesulfonate, $9 \mathrm{NaCl}$, 0.9 EGTA, 9 HEPES, 14 Tris-phosphocreatine, 2 Mg-ATP, 0.3 Tris-GTP, and $2 \mathrm{Na}_{2}$-ATP, $\mathrm{pH}$ adjusted to 7.3 with $\mathrm{KOH}$. The electrode solution was adjusted to 305 mosM with glucose, and bath osmolarity was 300 mosM. This internal solution supports BK current activation and spontaneous firing ${ }^{23,49}$. After $\mathrm{G} \Omega$ seal and whole-cell break-in, membrane properties were elicited from a $+20 \mathrm{mV}$ voltage step from a holding potential $\left(V_{\mathrm{h}}\right)$ of $-90 \mathrm{mV}$. Access resistance was verified to be $<25 \mathrm{M} \Omega$ with $< \pm 5 \%$ change at the end of the recording (on average $\sim 15 \mathrm{M} \Omega$ ). Series resistance was compensated at $80 \%$.

For action potentials, data were acquired in $10 \mathrm{~s}$ sweeps and filtered at $10 \mathrm{kHz}$. Frequency was calculated as the average of each sweep. Baseline potential was determined as the average inter-spike potential in the presence of spontaneous activity. Template-based action potential analysis was performed in Clampfit 10 (Molecular Devices) to obtain action potential peak and half-width $\left(t_{1 / 2}\right)$ values. Afterhyperpolarization (AHP) peak was determined as the peak negative value of the action potential, and AHP amplitude was the baseline membrane potential minus the AHP peak value. Threshold analysis was performed by taking the derivative of each action potential event in the $10 \mathrm{~s}$ segment to determine the inflection in the voltage trajectory (Clampfit). This method gives a set of values per neuron with lower standard deviation than automated analysis using $d V / d t$ of $4-5 \mathrm{~V} \mathrm{~s}^{-1}$ (ref. 49). Action potential analysis was performed blind with respect to genotype or time point.

In voltage-clamp mode, total voltage-activated $\mathrm{K}^{+}$currents were elicited from a holding potential of $-90 \mathrm{mV}$, stepping from -110 to $+90 \mathrm{mV}$ for either 30 or $150 \mathrm{~ms}$ in $20 \mathrm{mV}$ increments. BK currents were isolated by focal application of the BK antagonist paxilline $(10 \mu \mathrm{M})$, and subtracting currents after paxilline from the initial current. The effective concentration of paxilline in the recoding chamber was $3.75 \mu \mathrm{M}$. Currents were averaged from three to five voltage families Current-voltage $(I-V)$ relationships were constructed from the current level at $30 \mathrm{~ms}$ from each potential, unless noted otherwise (peak or steady-state values). Current density values were obtained by normalizing to cell capacitance, which did not differ between day and night (7.0 \pm 0.5 and $6.8 \pm 0.4 \mathrm{pF}$, respectively) or between WT and $\beta 2 \mathrm{KO}(7.7 \pm 0.7$ and $7.1 \pm 0.4 \mathrm{pF}, n=27,20$, respectively). Voltage values were adjusted for the liquid junction potential of $10 \mathrm{mV}$. $\tau_{\text {inact }}$ values were calculated from single exponential fits of the macroscopic current decay from the $+90 \mathrm{mV}$ step for $150 \mathrm{~ms}$ (Clampfit, Molecular Devices). $\mathrm{BK}_{\mathrm{i}}$ and $\mathrm{BK}_{\mathrm{s}}$ currents were categorized as described using the current ratio at $30 \mathrm{~ms}$ from $+90 \mathrm{mV}$ step $\left(\mathrm{BK}_{\mathrm{i}}<0.07, \mathrm{BK}_{\mathrm{s}}>0.07\right)$, corresponding to $\tau_{\text {inact }}$ values $<110 \mathrm{~ms}$.

For passive membrane properties, whole-cell recordings were performed as above using the same internal solution as for current- and voltage-clamp recording, with tetrodotoxin $(1 \mu \mathrm{M})$ in the bath. After break in, the membrane potential was recorded, followed by current injection steps ( 0 to $-25 \mathrm{pA}$, in $5 \mathrm{pA}$ increments). Input resistance was calculated as the linear slope of the voltage. For experiments where currents were categorized as $\mathrm{BK}_{\mathrm{s}}$ or $\mathrm{BK}_{\mathrm{i}}$, cells were switched to voltageclamp mode after recording $V_{\mathrm{m}}$ and $R_{\mathrm{i}}$, and BK currents were isolated and $\tau_{\text {inact }}$ values were obtained as described in the previous section. 
For $\mathrm{BK}_{\mathrm{i}}$ and $\mathrm{BK}_{\mathrm{s}}$ recordings of $V_{\mathrm{m}}, R_{\mathrm{i}}$, subthreshold $\mathrm{SCN} \mathrm{BK}$ currents, and $\mathrm{BK}$ currents evoked by action potential commands, recordings were performed in $1 \mu \mathrm{M}$ tetrodotoxin. After recording $V_{\mathrm{m}}$ and $R_{\mathrm{i}}$ as described above, cells were switched to voltage-clamp mode and stepped from a holding potential of -90 to $+90 \mathrm{mV}$ for $150 \mathrm{~ms}$ to determine $\mathrm{BK}_{\mathrm{i}}$ and $\mathrm{BK}_{\mathrm{s}}$ by $\tau_{\text {inact }}$ as described previously. After holding at $-150 \mathrm{mV}$ for $100 \mathrm{~ms}$, cells were stepped from -60 to $-30 \mathrm{mV}$ (in $5 \mathrm{mV}$ increments), and then stepped back to $-150 \mathrm{mV}$ for $100 \mathrm{~ms}$ followed by action potential commands. Previously acquired native action potential waveform was obtained from daytime or night time SCN recordings. For day (Fig. 10c) and night (Supplementary Fig. 7), the action potential parameters are indicated in the figure legends. Three sequenced action potentials were applied at the average $\mathrm{BK}_{\mathrm{i}}$ daytime firing rate $(2.25 \mathrm{~Hz})$ or night time $\mathrm{BK}_{\mathrm{s}}$ firing rate $(1 \mathrm{~Hz})$ as depicted in the figures. Current-voltage relationships were plotted, and the action potential-evoked peak and subthreshold currents were normalized to the cell capacitance.

Drugs and $\mathbf{\beta 2 N}$ peptides. Paxilline and tetrodotoxin (Tocris, Bristol, UK) were prepared as $1,000 \times$ stocks in DMSO and water, respectively. $\beta 2 \mathrm{~N}$, was synthesized as the first 45 amino acids of the $\beta 2$ subunit (MFIWTSGRTSSSYRQDEKRNIYQKIRDHDLLDKRKTVTALKAGED) (GenScript, Piscatawa, NJ, USA ${ }^{41}$. $\beta 2 \mathrm{~N}^{\Delta \mathrm{FIW}}$ was a 42 amino acid peptide with the underlined FIW deletion. Lyophilized peptides were reconstituted in intracellular solution as $100 \times$ stocks and added at the final indicated concentrations.

HEK cell patch-clamp electrophysiology. HEK293T cells (ATCC, Manassas, VA, USA) were transfected with the $\mathrm{BK}_{\mathrm{SRKR}} \alpha$ subunit cDNA (Genbank accession \#IX46275), with or without $\beta 2$ (ref. 34), both in pcDNA3.1. DNA was transfected at $1 \mu \mathrm{g}$ ( $\alpha$ only) or $1 \mu \mathrm{g} \alpha$ and $1.7 \mu \mathrm{g} \beta 2$ per $35 \mathrm{~mm}$ dish using TransIT (Mirus Bio, Madison, WI, USA). Cells were plated on glass coverslips $3-5 \mathrm{~h}$ later and recorded from 20 to $30 \mathrm{~h}$ after transfection. Patch-clamp recording was performed using the voltage-clamp mode in the inside-out patch configuration using thin-walled borosilicate pipettes with resistances of $2-5 \mathrm{M} \Omega$ as described previously ${ }^{60}$. Recordings were made at room temperature, and the data were acquired at $50 \mathrm{kHz}$ and filtered at $10 \mathrm{kHz}$. The bath (intracellular) solution was composed of $(\mathrm{mM}): 140 \mathrm{KMeSO}_{3}, 2$ $\mathrm{KCl}, 20$ HEPES and 5 HEDTA. Free $\left[\mathrm{Ca}^{2+}\right]_{\mathrm{i}}$ was calculated using Webmax $\mathrm{C}$ Standard software ${ }^{61}$, to a final concentration of $50 \mu \mathrm{M}$ (from $1 \mathrm{M} \mathrm{CaCl}_{2}$ stock). The pipette (extracellular) solution consisted of (mM): $145 \mathrm{NaCl}, 5 \mathrm{KCl}, 2 \mathrm{CaCl}_{2}, 1$ $\mathrm{MgCl} 2$ and 10 HEPES, with $\mathrm{pH}$ adjusted to 7.2 with $\mathrm{NaOH}$. Cells were held at $-150 \mathrm{mV}$, followed by a maximally activating test step to $+150 \mathrm{mV}$ for $150 \mathrm{~ms}$. Data were collected only from patches with $<0.7$ fractional current $\left(I_{\text {peak }} / I_{\mathrm{ss}}\right)$. BK currents were elicited from patches using the voltage protocols indicated in the figures. Single-exponential functions were fit to the macroscopic current decay at $+90 \mathrm{mV}$.

For activation of BK currents by action potential commands (Supplementary Fig. 8e), the daytime action potential waveform (as in Fig. 10c) was applied after the standard voltage protocol in whole-cell voltage-clamp mode. The pipette (intracellular) and bath (extracellular) solution were the same as used in the SCN whole recordings above. The action potential-evoked peak current, or the subthreshold current, was normalized to the maximal current elicited from each patch.

Statistics. The number of neurons (BK current and action potential recordings), the number of mice (circadian behaviour) or the number of SCN slice cultures (multielectrode array recordings) are represented as $n$. Group sample sizes were determined based on power calculations using effect sizes estimated from previous experiments. Behavioural comparisons were performed as comparisons between WT and $\beta 2 \mathrm{KO}$, or WT and $\beta 4 \mathrm{KO}$, using an unpaired Student's $t$-test. One-way ANOVAs were used for comparisons with more than two conditions, and the $P$ values reported in the text are the Bonferroni post hoc tests only for comparisons where the main effect was $P<0.05$. Factorial ANOVA was used for comparing multiple conditions across voltages, with Bonferroni post hoc $P$ values for specific comparisons reported in the text. Categorical data (number of rhythmic versus arrhythmic recordings, or $\mathrm{BK}_{\mathrm{i}}$ versus $\mathrm{BK}_{\mathrm{s}}$ ) were compared with Fisher's exact test, with the Fisher's $P$ value reported in the text. Statistical tests were run in Origin (Originlabs, Northampton, MA, USA). All values are presented as mean \pm s.e.m.

\section{References}

1. Hodgkin, A. L. \& Huxley, A. F. The dual effect of membrane potential on sodium conductance in the giant axon of Loligo. J. Physiol. 116, 497-506 (1952).

2. Armstrong, C. M. \& Bezanilla, F. Inactivation of the sodium channel II. Gating current experiments. J. Gen. Physiol. 70, 567-590 (1977).

3. Hoshi, T., Zagotta, W. N. \& Aldrich, R. W. Two types of inactivation in Shaker $\mathrm{K}^{+}$channels: effects of alterations in the carboxy-terminal region. Neuron 7, 547-556 (1991).

4. Zuhlke, R. D., Pitt, G. S., Deisseroth, K., Tsien, R. W. \& Reuter, H. Calmodulin supports both inactivation and facilitation of L-type calcium channels. Nature 399, 159-162 (1999).
5. Peterson, B. Z., DeMaria, C. D., Adelman, J. P. \& Yue, D. T. Calmodulin is the $\mathrm{Ca}^{2+}$ sensor for $\mathrm{Ca}^{2+}$-dependent inactivation of L-type calcium channels. Neuron 22, 549-558 (1999).

6. Hoshi, T., Zagotta, W. N. \& Aldrich, R. W. Biophysical and molecular mechanisms of Shaker potassium channel inactivation. Science 250, 533-538 (1990).

7. Wallner, M., Meera, P. \& Toro, L. Molecular basis of fast inactivation in voltage and $\mathrm{Ca}^{2+}$-activated $\mathrm{K}^{+}$channels: a transmembrane beta-subunit homolog. Proc. Natl. Acad. Sci. USA 96, 4137-4142 (1999).

8. Xia, X. M., Ding, J. P. \& Lingle, C. J. Molecular basis for the inactivation of $\mathrm{Ca}^{2+}$ - and voltage-dependent BK channels in adrenal chromaffin cells and rat insulinoma tumor cells. J. Neurosci. 19, 5255-5264 (1999).

9. Pyott, S. J., Glowatzki, E., Trimmer, J. S. \& Aldrich, R. W. Extrasynaptic localization of inactivating calcium-activated potassium channels in mouse inner hair cells. J. Neurosci. 24, 9469-9474 (2004).

10. Hicks, G. A. \& Marrion, N. V. $\mathrm{Ca}^{2+}$-dependent inactivation of large conductance $\mathrm{Ca}^{2+}$-activated $\mathrm{K}^{+}(\mathrm{BK})$ channels in rat hippocampal neurones produced by pore block from an associated particle. J. Physiol. 508, 721-734 (1998).

11. Armstrong, C. E. \& Roberts, W. M. Rapidly inactivating and non-inactivating calcium-activated potassium currents in frog saccular hair cells. J. Physiol. 536, 49-65 (2001).

12. Li, W. et al. Characterization of voltage-and $\mathrm{Ca}^{2+}$-activated $\mathrm{K}^{+}$ channels in rat dorsal root ganglion neurons. J. Cell. Physiol. 212, 348-357 (2007).

13. Li, Z. W., Ding, J. P., Kalyanaraman, V. \& Lingle, C. J. RINm5f cells express inactivating BK channels whereas HIT cells express noninactivating BK channels. J. Neurophysiol. 81, 611-624 (1999).

14. Grimes, W. N., Li, W., Chavez, A. E. \& Diamond, J. S. BK channels modulate pre- and postsynaptic signaling at reciprocal synapses in retina. Nat. Neurosci. 12, 585-592 (2009).

15. Shao, L. R., Halvorsrud, R., Borg-Graham, L. \& Storm, J. F. The role of BK-type $\mathrm{Ca}^{2+}$-dependent $\mathrm{K}^{+}$channels in spike broadening during repetitive firing in rat hippocampal pyramidal cells. J. Physiol. 521, 135-146 (1999).

16. Abdul-Ghani, M. A., Valiante, T. A., Carlen, P. L. \& Pennefather, P. S. Tyrosine kinase inhibitors enhance a $\mathrm{Ca}^{2+}$-activated $\mathrm{K}^{+}$current (IAHP) and reduce IAHP suppression by a metabotropic glutamate receptor agonist in rat dentate granule neurones. J. Physiol. 496(Pt 1): 139-144 (1996).

17. Faber, E. S. \& Sah, P. Ca ${ }^{2+}$-activated $\mathrm{K}^{+}$(BK) channel inactivation contributes to spike broadening during repetitive firing in the rat lateral amygdala. J. Physiol. 552, 483-497 (2003).

18. Martinez-Espinosa, P. L., Yang, C., Gonzalez-Perez, V., Xia, X. M. \& Lingle, C. J. Knockout of the BK beta2 subunit abolishes inactivation of BK currents in mouse adrenal chromaffin cells and results in slow-wave burst activity. J. Gen. Physiol. 144, 275-295 (2014).

19. Solaro, C. R. \& Lingle, C. J. Trypsin-sensitive, rapid inactivation of a calciumactivated potassium channel. Science 257, 1694-1698 (1992).

20. Solaro, C. R., Prakriya, M., Ding, J. P. \& Lingle, C. J. Inactivating and noninactivating $\mathrm{Ca}^{2+}$ - and voltage-dependent $\mathrm{K}^{+}$current in rat adrenal chromaffin cells. J. Neurosci. 15, 6110-6123 (1995).

21. Sun, L. et al. Differential regulation of action potentials by inactivating and noninactivating BK channels in rat adrenal chromaffin cells. Biophys. J. 97, 1832-1842 (2009).

22. Montgomery, J. R. \& Meredith, A. L. Genetic activation of BK currents in vivo generates bidirectional effects on neuronal excitability. Proc. Natl. Acad. Sci. USA 109, 18997-19002 (2012).

23. Montgomery, J. R., Whitt, J. P., Wright, B. N., Lai, M. H. \& Meredith, A. L. Mis-expression of the $\mathrm{BK} \mathrm{K}^{+}$channel disrupts suprachiasmatic nucleus circuit rhythmicity and alters clock-controlled behavior. Am. J. Physiol. Cell Physiol. 304, C299-C311 (2013).

24. Meredith, A. L. et al. BK calcium-activated potassium channels regulate circadian behavioral rhythms and pacemaker output. Nat. Neurosci. 9, 1041-1049 (2006).

25. Kent, J. \& Meredith, A. L. BK channels regulate spontaneous action potential rhythmicity in the suprachiasmatic nucleus. PloS ONE 3, e3884 (2008).

26. Colwell, C. S. Linking neural activity and molecular oscillations in the SCN. Nat. Rev. Neurosci. 12, 553-569 (2011).

27. Low-Zeddies, S. S. \& Takahashi, J. S. Chimera analysis of the Clock mutation in mice shows that complex cellular integration determines circadian behavior. Cell 105, 25-42 (2001).

28. Liu, C., Weaver, D. R., Strogatz, S. H. \& Reppert, S. M. Cellular construction of a circadian clock: period determination in the suprachiasmatic nuclei. Cell 91, 855-860 (1997).

29. Ceriani, M. F. et al. Genome-wide expression analysis in Drosophila reveals genes controlling circadian behavior. J. Neurosci. 22, 9305-9319 (2002).

30. Panda, S. et al. Coordinated transcription of key pathways in the mouse by the circadian clock. Cell 109, 307-320 (2002). 
31. Pitts, G. R., Ohta, H. \& McMahon, D. G. Daily rhythmicity of largeconductance $\mathrm{Ca}^{2+}$-activated $\mathrm{K}^{+}$currents in suprachiasmatic nucleus neurons. Brain Res. 1071, 54-62 (2006).

32. Reppert, S. M. \& Weaver, D. R. Coordination of circadian timing in mammals. Nature 418, 935-941 (2002)

33. Farajnia, S., Meijer, J. H. \& Michel, S. Age-related changes in large-conductance calcium-activated potassium channels in mammalian circadian clock neurons. Neurobiol. Aging 36, 2176-2183 (2015).

34. Brenner, R., Jegla, T. J., Wickenden, A., Liu, Y. \& Aldrich, R. W. Cloning and functional characterization of novel large conductance calcium-activated potassium channel beta subunits, hKCNMB3 and hKCNMB4. J. Biol. Chem. 275, 6453-6461 (2000).

35. Aton, S. J., Colwell, C. S., Harmar, A. J., Waschek, J. \& Herzog, E. D. Vasoactive intestinal polypeptide mediates circadian rhythmicity and synchrony in mammalian clock neurons. Nat. Neurosci. 8, 476-483 (2005).

36. Sokolove, P. G. \& Bushell, W. N. The chi square periodogram: its utility for analysis of circadian rhythms. J. Theor. Biol. 72, 131-160 (1978).

37. Pendergast, J. S., Friday, R. C. \& Yamazaki, S. Photic entrainment of period mutant mice is predicted from their phase response curves. J. Neurosci. 30, 12179-12184 (2010).

38. Vitaterna, M. H. et al. The mouse Clock mutation reduces circadian pacemaker amplitude and enhances efficacy of resetting stimuli and phase-response curve amplitude. Proc. Natl. Acad. Sci. USA 103, 9327-9332 (2006).

39. Uebele, V. N. et al. Cloning and functional expression of two families of beta-subunits of the large conductance calcium-activated $\mathrm{K}^{+}$channel. J. Biol. Chem. 275, 23211-23218 (2000).

40. Sun, X., Yao, H., Zhou, D., Gu, X. \& Haddad, G. G. Modulation of hSlo BK current inactivation by fatty acid esters of CoA. J. Neurochem. 104, 1394-1403 (2008).

41. Bentrop, D., Beyermann, M., Wissmann, R. \& Fakler, B. NMR structure of the 'ball-and-chain' domain of KCNMB2, the beta2 subunit of large conductance $\mathrm{Ca}^{2+}$ - and voltage-activated potassium channels. J. Biol. Chem. 276, 42116-42121 (2001).

42. Xia, X. M., Ding, J. P. \& Lingle, C. J. Inactivation of BK channels by the NH2 terminus of the beta2 auxiliary subunit: an essential role of a terminal peptide segment of three hydrophobic residues. J. Gen. Physiol. 121, 125-148 (2003).

43. Cloues, R. K. \& Sather, W. A. Afterhyperpolarization regulates firing rate in neurons of the suprachiasmatic nucleus. J. Neurosci. 23, 1593-1604 (2003).

44. Colwell, C. S. BK channels and circadian output. Nat. Neurosci. 9, 985-986 (2006).

45. Kuhlman, S. J. \& McMahon, D. G. Rhythmic regulation of membrane potential and potassium current persists in SCN neurons in the absence of environmental input. Eur. J. Neurosci. 20, 1113-1117 (2004).

46. Wang, T. A. et al. Circadian rhythm of redox state regulates excitability in suprachiasmatic nucleus neurons. Science 337, 839-842 (2012).

47. Belle, M. D., Diekman, C. O., Forger, D. B. \& Piggins, H. D. Daily electrical silencing in the mammalian circadian clock. Science 326, 281-284 (2009).

48. de Jeu, M., Hermes, M. \& Pennartz, C. Circadian modulation of membrane properties in slices of rat suprachiasmatic nucleus. Neuroreport 9, 3725-3729 (1998).

49. Jackson, A. C., Yao, G. L. \& Bean, B. P. Mechanism of spontaneous firing in dorsomedial suprachiasmatic nucleus neurons. J. Neurosci. 24, 7985-7998 (2004).

50. Sheeba, V., Gu, H., Sharma, V. K., O’Dowd, D. K. \& Holmes, T. C. Circadian- and light-dependent regulation of resting membrane potential and spontaneous action potential firing of Drosophila circadian pacemaker neurons. J. Neurophysiol. 99, 976-988 (2008)

51. Flourakis, M. et al. A conserved bicycle model for circadian clock control of membrane excitability. Cell 162, 836-848 (2015).
52. Itri, J. N., Michel, S., Vansteensel, M. J., Meijer, J. H. \& Colwell, C. S. Fast delayed rectifier potassium current is required for circadian neural activity. Nat. Neurosci. 8, 650-656 (2005).

53. Brenner, R. Knockout of the BK beta2 subunit reveals the importance of accessorizing your channel. J. Gen. Physiol. 144, 351-356 (2014).

54. Pennartz, C. M., de Jeu, M. T., Bos, N. P., Schaap, J. \& Geurtsen, A. M. Diurnal modulation of pacemaker potentials and calcium current in the mammalian circadian clock. Nature 416, 286-290 (2002).

55. Ding, J. P. \& Lingle, C. J. Steady-state and closed-state inactivation properties of inactivating BK channels. Biophys. J. 82, 2448-2465 (2002).

56. Lancaster, B. \& Nicoll, R. A. Properties of two calcium-activated hyperpolarizations in rat hippocampal neurones. J. Physiol. 389, 187-203 (1987).

57. Storm, J. F. Action potential repolarization and a fast after-hyperpolarization in rat hippocampal pyramidal cells. J. Physiol. 385, 733-759 (1987).

58. Adams, P. R., Constanti, A., Brown, D. A. \& Clark, R. B. Intracellular $\mathrm{Ca}^{2+}$ activates a fast voltage-sensitive $\mathrm{K}^{+}$current in vertebrate sympathetic neurones. Nature 296, 746-749 (1982)

59. Brenner, R. et al. BK channel beta4 subunit reduces dentate gyrus excitability and protects against temporal lobe seizures. Nat. Neurosci. 8, 1752-1759 (2005).

60. Shelley, C., Whitt, J. P., Montgomery, J. R. \& Meredith, A. L. Phosphorylation of a constitutive serine inhibits $\mathrm{BK}$ channel variants containing the alternate exon 'SRKR'. J. Gen. Physiol. 142, 585-598 (2013).

61. Bers, D. M., Patton, C. W. \& Nuccitelli, R. A practical guide to the preparation of $\mathrm{Ca}^{2+}$ buffers. Methods Cell Biol. 99, 1-26 (2010).

\section{Acknowledgements}

We thank Chris Lingle for providing $\beta 2 \mathrm{KO}$ mice and critical discussions, and Brad Rothberg and Jon Lederer for comments on the manuscript. This work was supported by National Heart, Lung, and Blood Institute Grant HL102758 (ALM), The American Physiological Society's Ryuji Ueno Award, sponsored by the S\&R Foundation (ALM), and National Institute of Arthritis and Musculoskeletal and Skin Diseases Training Grant T32-AR007592 (JPW)

\section{Author contributions}

J.P.W. and A.L.M. designed the study. J.P.W. performed the experiments and statistical analysis. J.P.W. and A.L.M. analysed the data. J.R.M. analysed action potential data and performed statistical analysis. A.L.M. wrote the manuscript. All authors approved the final manuscript.

\section{Additional information}

Supplementary Information accompanies this paper at http://www.nature.com/ naturecommunications

Competing financial interests: The authors declare no competing financial interests.

Reprints and permission information is available online at http://npg.nature.com/ reprintsandpermissions/

How to cite this article: Whitt, J. P. et al. BK channel inactivation gates daytime excitability in the circadian clock. Nat. Commun. 7:10837 doi: 10.1038/ncomms10837 (2016).

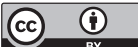

This work is licensed under a Creative Commons Attribution 4.0 International License. The images or other third party material in this article are included in the article's Creative Commons license, unless indicated otherwise in the credit line; if the material is not included under the Creative Commons license, users will need to obtain permission from the license holder to reproduce the material To view a copy of this license, visit http://creativecommons.org/licenses/by/4.0/ 